\title{
Measurement of heavy-quark jet photoproduction at HERA
}

\author{
The ZEUS Collaboration
}

H. Abramowicz ${ }^{45, b c}$, I. Abt ${ }^{35}$, L. Adamczyk ${ }^{13}$, M. Adamus ${ }^{54}$, R. Aggarwal $^{7, x}$, S. Antonelli ${ }^{4}$, P. Antonioli ${ }^{3}$, A. Antonov ${ }^{33}$, M. Arneodo ${ }^{50}$, V. Aushev ${ }^{26,27, \text { av }}$, Y. Aushev ${ }^{27, \text { aw }}$, O. Bachynska ${ }^{15}$, A. Bamberger ${ }^{19}$, A.N. Barakbaev ${ }^{25}$, G. Barbagli $^{17}$, G. Bari ${ }^{3}$, F. Barreiro ${ }^{30}$, N. Bartosik ${ }^{27, \text { ax }}$, D. Bartsch ${ }^{5}$, M. Basile ${ }^{4}$, O. Behnke $^{15}$, J. Behr ${ }^{15}$, U. Behrens ${ }^{15}$, L. Bellagamba ${ }^{3}$, A. Bertolin ${ }^{39}$, S. Bhadra ${ }^{57}$, M. Bindi ${ }^{4}$, C. Blohm ${ }^{15}$, V. Bokhonov ${ }^{26, \text { av }}$, T. Bołd ${ }^{13}$, O. Bolilyi ${ }^{27, \text { ax }}$, K. Bondarenko ${ }^{27}$, E.G. Boos ${ }^{25}$, K. Borras ${ }^{15}$, D. Boscherini ${ }^{3}$, D. Bot ${ }^{15}$, I. Brock ${ }^{5}$, E. Brownson ${ }^{56}$, R. Brugnera ${ }^{40}$, N. Brümmer ${ }^{37}$, A. Bruni ${ }^{3}$, G. Bruni ${ }^{3}$, B. Brzozowska ${ }^{53}$, P.J. Bussey ${ }^{20}$, B. Bylsma ${ }^{37}$, A. Caldwell ${ }^{35}$, M. Capua $^{8}$, R. Carlin $^{40}$, C.D. Catterall ${ }^{57}$, S. Chekanov ${ }^{1}$, J. Chwastowski ${ }^{12, z}$, J. Ciborowski ${ }^{53, \text { bg }}$, R. Ciesielski ${ }^{15, a b}$, L. Cifarelli ${ }^{4}$, F. Cindolo ${ }^{3}$, A. Contin ${ }^{4}$, A.M. Cooper-Sarkar ${ }^{38}$, N. Coppola ${ }^{15, a c}$, M. Corradi ${ }^{3}$, F. Corriveau ${ }^{31}$, M. Costa ${ }^{49}$,

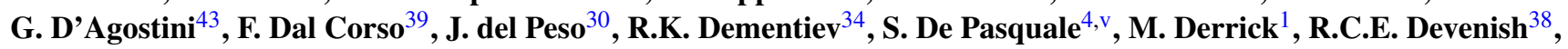
D. Dobur ${ }^{19, a 0}$, B.A. Dolgoshein ${ }^{33, b}$, G. Dolinska ${ }^{26,27}$, A.T. Doyle ${ }^{20}$, V. Drugakov ${ }^{16}$, L.S. Durkin ${ }^{37}$, S. Dusini ${ }^{39}$, Y. Eisenberg ${ }^{55}$, P.F. Ermolov ${ }^{34, b}$, A. Eskreys ${ }^{12, b}$, S. Fang ${ }^{15, \text { ad }}$, S. Fazio ${ }^{8}$, J. Ferrando ${ }^{38}$, M.I. Ferrero ${ }^{49}$, J. Figiel ${ }^{12}$, M. Forrest ${ }^{20, a r}$, B. Foster ${ }^{38}$, S. Fourletov ${ }^{51, a q}$, G. Gach ${ }^{13}$, A. Galas ${ }^{12}$, E. Gallo ${ }^{17}$, A. Garfagnini ${ }^{40}$, A. Geiser ${ }^{15}$, I. Gialas ${ }^{21, \text { as }}$, L.K. Gladilin ${ }^{34}$, D. Gladkov ${ }^{33}$, C. Glasman ${ }^{30}$, O. Gogota ${ }^{26,27}$, Yu.A. Golubkov ${ }^{34}$, P. Göttlicher ${ }^{15, \text { ae }}$, I. Grabowska-Bołd ${ }^{13}$, J. Grebenyuk ${ }^{15}$, I. Gregor ${ }^{15}$, G. Grigorescu ${ }^{36}$, G. Grzelak ${ }^{53}$, O. Gueta ${ }^{45}$, C. Gwenlan ${ }^{38, a z}$,

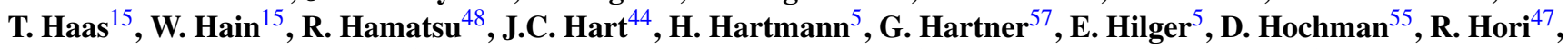
K. Horton ${ }^{38, \text { ba }}$, A. Hüttmann ${ }^{15}$, Z.A. Ibrahim ${ }^{10}$, Y. Iga $^{42}$, R. Ingbir ${ }^{45}$, M. Ishitsuka ${ }^{46}$, H.-P. Jakob ${ }^{5}$, F. Januschek ${ }^{15}$, M. Jimenez ${ }^{30}$, T.W. Jones ${ }^{52}$, M. Jüngst ${ }^{5}$, I. Kadenko ${ }^{27}$, B. Kahle ${ }^{15}$, B. Kamaluddin ${ }^{10, b}$, S. Kananov ${ }^{45}$, T. Kanno ${ }^{46}$, U. Karshon ${ }^{55}$, F. Karstens ${ }^{19, \text { ap }}$, I.I. Katkov ${ }^{15, a f}$, M. Kaur ${ }^{7}$, P. Kaur ${ }^{7, x}$, A. Keramidas $^{36}$, L.A. Khein ${ }^{34}$, J.Y. Kim ${ }^{9}$, D. Kisielewska $^{13}$, S. Kitamura ${ }^{48, \text { be }}$, R. Klanner ${ }^{22}$, U. Klein ${ }^{15, \text { ag }}$, E. Koffeman ${ }^{36}$, P. Kooijman ${ }^{36}$, I. Korol ${ }^{26,27}$, I.A. Korzhavina ${ }^{34}$, A. Kotański ${ }^{14, \text { aa }}$, U. Kötz ${ }^{15}$, H. Kowalski ${ }^{15}$, P. Kulinski ${ }^{53}$, O. Kuprash ${ }^{27, \text { ay }}$, M. Kuze ${ }^{46}$, A. Lee Le $^{37}$, B.B. Levchenko ${ }^{34}$, A. Levy ${ }^{45, a}$, V. Libov ${ }^{15}$, S. Limentani ${ }^{40}$, T.Y. Ling $^{37}$, M. Lisovyi ${ }^{15}$, E. Lobodzinska ${ }^{15}$, W. Lohmann ${ }^{16}$, B. Löhr ${ }^{15}$, E. Lohrmann ${ }^{22}$, K.R. Long ${ }^{23}$, A. Longhin ${ }^{39}$, D. Lontkovskyi ${ }^{27, \text { ay }}$, O.Yu. Lukina ${ }^{34}$, P. Łużniak ${ }^{53, \text { bh }}$, J. Maeda ${ }^{46, b d}$, S. Magill ${ }^{1}$, I. Makarenko ${ }^{27, \text { ay }}$, J. Malka ${ }^{53, \text { bh }}$, R. Mankel ${ }^{15}$, A. Margotti ${ }^{3}$, G. Marini $^{43}$, J.F. Martin ${ }^{51}$, A. Mastroberardino ${ }^{8}$, M.C.K. Mattingly ${ }^{2}$, I.-A. Melzer-Pellmann ${ }^{15}$, S. Mergelmeyer ${ }^{5}$,

S. Miglioranzi ${ }^{15, \text { ah }}$, F. Mohamad Idris ${ }^{10}$, V. Monaco $^{49}$, A. Montanari ${ }^{15}$, J.D. Morris ${ }^{6, w}$, K. Mujkic ${ }^{15, a i}$, B. Musgrave ${ }^{1}$, K. Nagano ${ }^{24}$, T. Namsoo ${ }^{15, \text { aj }}$, R. Nania $^{3}$, D. Nicholass ${ }^{1, u}$, A. Nigro ${ }^{43}$, Y. Ning ${ }^{11}$, T. Nobe ${ }^{46}$, U. Noor ${ }^{57}$, D. Notz ${ }^{15}$, R.J. Nowak ${ }^{53}$, A.E. Nuncio-Quiroz ${ }^{5}$, B.Y. Oh $^{41}$, N. Okazaki ${ }^{47}$, K. Oliver $^{38}$, K. Olkiewicz ${ }^{12}$, Yu. Onishchuk ${ }^{27}$, K. Papageorgiu ${ }^{21}$, A. Parenti ${ }^{15}$, E. Paul ${ }^{5}$, J.M. Pawlak ${ }^{53}$, B. Pawlik ${ }^{12}$, P.G. Pelfer ${ }^{18}$, A. Pellegrino ${ }^{36}$, W. Perlanski ${ }^{53, \text { bh }}$, H. Perrey ${ }^{22}$, K. Piotrzkowski ${ }^{29}$, P. Plucinski ${ }^{54, b i}$, N.S. Pokrovskiy ${ }^{25}$, A. Polini ${ }^{3}$, A.S. Proskuryakov ${ }^{34}$, M. Przybycień ${ }^{13}$, A. Raval ${ }^{15}$, D.D. Reeder ${ }^{56}$, B. Reisert $^{35}$, Z. $\operatorname{Ren}^{11}$, J. Repond ${ }^{1}$, Y.D. Ri ${ }^{48, b f}$, A. Robertson ${ }^{38}$, P. Roloff $^{15}$, E. Ron $^{30}$, I. Rubinsky ${ }^{15}$, M. Ruspa ${ }^{50}$, R. Sacchi ${ }^{49}$, A. Salii ${ }^{27}$, U. Samson ${ }^{5}$, G. Sartorelli ${ }^{4}$, A.A. Savin ${ }^{56}$, D.H. Saxon ${ }^{20}$, M. Schioppa ${ }^{8}$, S. Schlenstedt ${ }^{16}$, P. Schleper ${ }^{22}$, W.B. Schmidke ${ }^{35}$, U. Schneekloth ${ }^{15}$, V. Schönberg ${ }^{5}$, T. Schörner-Sadenius ${ }^{15}$, J. Schwartz ${ }^{31}$, F. Sciulli ${ }^{11}$, L.M. Shcheglova ${ }^{34}$, R. Shehzadi ${ }^{5}$, S. Shimizu ${ }^{47, \text { ah, }}$ I. Singh ${ }^{7, x}$, I.O. Skillicorn ${ }^{20}$, W. Słomiński ${ }^{14}$, W.H. Smith ${ }^{56}$, V. Sola ${ }^{49}$, A. Solano ${ }^{49}$, D. Son ${ }^{28}$, V. Sosnovtsev ${ }^{33}$,

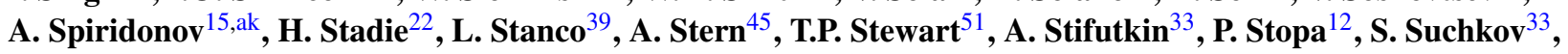
G. Susinno $^{8}$, L. Suszycki ${ }^{13}$, J. Sztuk-Dambietz ${ }^{22}$, D. Szuba ${ }^{15, a 1}$, J. Szuba' ${ }^{15, a m}$, A.D. Tapper $^{23}$, E. Tassi ${ }^{8, y}$, J. Terrón $^{30}$, T. Theedt ${ }^{15}$, H. Tiecke ${ }^{36}$, K. Tokushuku ${ }^{24, \text { at }}$, O. Tomalak ${ }^{27}$, J. Tomaszewska ${ }^{15, \text { an }}$, T. Tsurugai ${ }^{32}$, M. Turcato ${ }^{22}$,

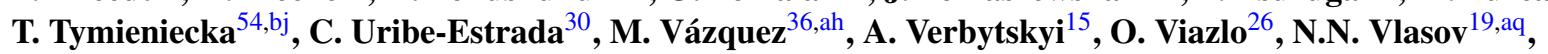
O. Volynets $^{27}$, R. Walczak ${ }^{38}$, W.A.T. Wan Abdullah ${ }^{10}$, J.J. Whitmore ${ }^{41, b b}$, J. Whyte ${ }^{57}$, L. Wiggers ${ }^{36}$, M. Wing ${ }^{52}$, M. Wlasenko ${ }^{5}$, G. Wolf ${ }^{15}$, H. Wolfe ${ }^{56}$, K. Wrona ${ }^{15}$, A.G. Yagües-Molina ${ }^{15}$, S. Yamada ${ }^{24}$, Y. Yamazaki ${ }^{24, a u}$, R. Yoshida $^{1}$, C. Youngman ${ }^{15}$, A.F. Żarnecki ${ }^{53}$, L. Zawiejski ${ }^{12}$, O. Zenaiev $^{27}$, W. Zeuner ${ }^{15, \text { ah }}$, B.O. Zhautykov ${ }^{25}$, N. Zhmak ${ }^{26, a v}$, C. Zhou $^{31}$, A. Zichichi ${ }^{4}$, M. Zolko ${ }^{27}$, D.S. Zotkin ${ }^{34}$, Z. Zulkapli1 ${ }^{10}$ 
${ }^{1}$ Argonne National Laboratory, Argonne, IL 60439-4815, USA ${ }^{c}$

${ }^{2}$ Andrews University, Berrien Springs, MI 49104-0380, USA

${ }^{3}$ INFN Bologna, Bologna, Italy ${ }^{\mathrm{d}}$

${ }^{4}$ University and INFN Bologna, Bologna, Italy ${ }^{\mathrm{d}}$

${ }^{5}$ Physikalisches Institut der Universität Bonn, Bonn, Germany ${ }^{\mathrm{e}}$

${ }^{6}$ H.H. Wills Physics Laboratory, University of Bristol, Bristol, $\mathrm{UK}^{\mathrm{f}}$

${ }^{7}$ Department of Physics, Panjab University, Chandigarh, India

${ }^{8}$ Physics Department and INFN, Calabria University, Cosenza, Italy ${ }^{\mathrm{d}}$

${ }_{9}^{9}$ Institute for Universe and Elementary Particles, Chonnam National University, Kwangju, South Korea

${ }^{10}$ Jabatan Fizik, Universiti Malaya, 50603 Kuala Lumpur, Malaysiag $^{\mathrm{g}}$

${ }^{11}$ Nevis Laboratories, Columbia University, Irvington on Hudson, NY 10027, USA ${ }^{\mathrm{h}}$

${ }^{12}$ The Henryk Niewodniczanski Institute of Nuclear Physics, Polish Academy of Sciences, Cracow, Poland ${ }^{\mathrm{i}}$

${ }^{13}$ Faculty of Physics and Applied Computer Science, AGH-University of Science and Technology, Cracow, Poland ${ }^{\mathrm{j}}$

${ }^{14}$ Department of Physics, Jagellonian University, Cracow, Poland

${ }^{15}$ Deutsches Elektronen-Synchrotron DESY, Hamburg, Germany

${ }^{16}$ Deutsches Elektronen-Synchrotron DESY, Zeuthen, Germany

${ }^{17}$ INFN Florence, Florence, Italy ${ }^{\mathrm{d}}$

${ }^{18}$ University and INFN Florence, Florence, Italy ${ }^{\mathrm{d}}$

${ }^{19}$ Fakultät für Physik der Universität Freiburg i.Br., Freiburg i.Br., Germany

${ }^{20}$ School of Physics and Astronomy, University of Glasgow, Glasgow, $\mathrm{UK}^{\mathrm{f}}$

${ }^{21}$ Department of Engineering in Management and Finance, Univ. of the Aegean, Chios, Greece

${ }^{22}$ Institute of Experimental Physics, Hamburg University, Hamburg, Germanyk

${ }^{23}$ High Energy Nuclear Physics Group, Imperial College London, London, UK ${ }^{\mathrm{f}}$

${ }^{24}$ Institute of Particle and Nuclear Studies, KEK, Tsukuba, Japan ${ }^{1}$

${ }^{25}$ Institute of Physics and Technology of Ministry of Education and Science of Kazakhstan, Almaty, Kazakhstan

${ }^{26}$ Institute for Nuclear Research, National Academy of Sciences, Kyiv, Ukraine

${ }^{27}$ Department of Nuclear Physics, National Taras Shevchenko University of Kyiv, Kyiv, Ukraine

${ }^{28}$ Center for High Energy Physics, Kyungpook National University, Daegu, South Korea ${ }^{\mathrm{m}}$

${ }^{29}$ Institut de Physique Nucléaire, Université Catholique de Louvain, Louvain-la-Neuve, Belgium ${ }^{\mathrm{n}}$

${ }^{30}$ Departamento de Física Teórica, Universidad Autónoma de Madrid, Madrid, Spain ${ }^{\circ}$

${ }^{31}$ Department of Physics, McGill University, Montréal, Québec, Canada H3A 2T8p

${ }^{32}$ Faculty of General Education, Meiji Gakuin University, Yokohama, Japan ${ }^{1}$

${ }^{33}$ Moscow Engineering Physics Institute, Moscow, Russia ${ }^{\mathrm{q}}$

${ }^{34}$ Institute of Nuclear Physics, Moscow State University, Moscow, Russia ${ }^{\mathrm{r}}$

${ }^{35}$ Max-Planck-Institut für Physik, München, Germany

${ }^{36}$ NIKHEF and University of Amsterdam, Amsterdam, Netherlands ${ }^{\mathrm{s}}$

${ }^{37}$ Physics Department, Ohio State University, Columbus, OH 43210, USA ${ }^{\mathrm{c}}$

${ }^{38}$ Department of Physics, University of Oxford, Oxford, UK $^{\mathrm{f}}$

${ }^{39}$ INFN Padova, Padova, Italy ${ }^{\mathrm{d}}$

${ }^{40}$ Dipartimento di Fisica dell' Università and INFN, Padova, Italy ${ }^{\mathrm{d}}$

${ }^{41}$ Department of Physics, Pennsylvania State University, University Park, PA 16802, USA ${ }^{\text {h }}$

${ }^{42}$ Polytechnic University, Sagamihara, Japan ${ }^{1}$

${ }^{43}$ Dipartimento di Fisica, Università 'La Sapienza' and INFN, Rome, Italy ${ }^{\mathrm{d}}$

${ }^{44}$ Rutherford Appleton Laboratory, Chilton, Didcot, Oxon, UK ${ }^{\mathrm{f}}$

${ }^{45}$ Raymond and Beverly Sackler Faculty of Exact Sciences, School of Physics, Tel Aviv University, Tel Aviv, Israel ${ }^{t}$

${ }^{46}$ Department of Physics, Tokyo Institute of Technology, Tokyo, Japan ${ }^{1}$

${ }^{47}$ Department of Physics, University of Tokyo, Tokyo, Japan ${ }^{1}$

${ }^{48}$ Department of Physics, Tokyo Metropolitan University, Tokyo, Japan ${ }^{1}$

${ }^{49}$ Università di Torino and INFN, Torino, Italy ${ }^{\mathrm{d}}$

${ }^{50}$ Università del Piemonte Orientale, Novara, and INFN, Torino, Italy ${ }^{\mathrm{d}}$

${ }^{51}$ Department of Physics, University of Toronto, Toronto, Ontario, Canada M5S 1A7 ${ }^{\mathrm{P}}$

${ }^{52}$ Physics and Astronomy Department, University College London, London, UK

${ }^{53}$ Faculty of Physics, University of Warsaw, Warsaw, Poland

${ }^{54}$ Institute for Nuclear Studies, Warsaw, Poland

${ }^{55}$ Department of Particle Physics and Astrophysics, Weizmann Institute, Rehovot, Israel

${ }^{56}$ Department of Physics, University of Wisconsin, Madison, WI 53706, USA ${ }^{\mathrm{c}}$

${ }^{57}$ Department of Physics, York University, Ontario, Canada M3J 1P3 ${ }^{\mathrm{p}}$

Received: 28 April 2011 / Revised: 10 May 2011 / Published online: 28 May 2011

(C) The Author(s) 2011. This article is published with open access at Springerlink.com 
Abstract Photoproduction of beauty and charm quarks in events with at least two jets has been measured with the ZEUS detector at HERA using an integrated luminosity of $133 \mathrm{pb}^{-1}$. The fractions of jets containing $b$ and $c$ quarks were extracted using the invariant mass of charged tracks associated with secondary vertices and the decay-length significance of these vertices. Differential cross sections as a function of jet transverse momentum, $p_{\mathrm{T}}^{\mathrm{jet}}$, and pseudora-

a e-mail: levy@alzt.tau.ac.il

${ }^{b}$ Deceased.

${ }^{\mathrm{c}}$ Supported by the US Department of Energy.

${ }^{\mathrm{d}}$ Supported by the Italian National Institute for Nuclear Physics (INFN).

${ }^{\text {e}}$ Supported by the German Federal Ministry for Education and Research (BMBF), under contract No. 05 H09PDF.

${ }^{\mathrm{f}}$ Supported by the Science and Technology Facilities Council, UK.

${ }^{g}$ Supported by an FRGS grant from the Malaysian government.

${ }^{\mathrm{h}}$ Supported by the US National Science Foundation. Any opinion, findings and conclusions or recommendations expressed in this material are those of the authors and do not necessarily reflect the views of the National Science Foundation.

${ }^{i}$ Supported by the Polish Ministry of Science and Higher Education as a scientific project No. DPN/N188/DESY/2009.

jSupported by the Polish Ministry of Science and Higher Education as a scientific project (2009-2010).

${ }^{\mathrm{k}}$ Supported by the German Federal Ministry for Education and Research (BMBF), under contract No. 05h09GUF, and the SFB 676 of the Deutsche Forschungsgemeinschaft (DFG).

${ }^{1}$ Supported by the Japanese Ministry of Education, Culture, Sports, Science and Technology (MEXT) and its grants for Scientific Research.

${ }^{\mathrm{m}}$ Supported by the Korean Ministry of Education and Korea Science and Engineering Foundation.

${ }^{\mathrm{n}}$ Supported by FNRS and its associated funds (IISN and FRIA) and by an Inter-University Attraction Poles Programme subsidised by the Belgian Federal Science Policy Office.

${ }^{o}$ Supported by the Spanish Ministry of Education and Science through funds provided by CICYT.

${ }^{\mathrm{p}}$ Supported by the Natural Sciences and Engineering Research Council of Canada (NSERC).

${ }^{\mathrm{q}}$ Partially supported by the German Federal Ministry for Education and Research (BMBF).

${ }^{\mathrm{r}}$ Supported by RF Presidential grant N 41-42.2010.2 for the Leading Scientific Schools and by the Russian Ministry of Education and Science through its grant for Scientific Research on High Energy Physics.

${ }^{\mathrm{s}}$ Supported by the Netherlands Foundation for Research on Matter (FOM).

${ }^{\mathrm{t}}$ Supported by the Israel Science Foundation.

u Also affiliated with University College London, United Kingdom.

${ }^{\mathrm{v}}$ Now at University of Salerno, Italy.

${ }^{w}$ Now at Queen Mary University of London, United Kingdom.

${ }^{\mathrm{x}}$ Also funded by Max Planck Institute for Physics, Munich, Germany

y Also Senior Alexander von Humboldt Research Fellow at Hamburg University, Institute of Experimental Physics, Hamburg, Germany.

${ }^{\mathrm{z}}$ Also at Cracow University of Technology, Faculty of Physics, Mathematics and Applied Computer Science, Poland pidity, $\eta^{\text {jet }}$, were measured. The data are compared with previous measurements and are well described by next-toleading-order QCD predictions.

\section{Introduction}

The study of beauty and charm production in $e p$ collisions constitutes a rigorous test of perturbative Quantum Chro-

\footnotetext{
${ }^{\text {aa }}$ Supported by the research grant No. 1 P03B 04529 (2005-2008).

${ }^{\mathrm{ab}}$ Now at Rockefeller University, New York, NY 10065, USA.

${ }^{a c}$ Now at DESY group FS-CFEL-1.

${ }^{a d}$ Now at Institute of High Energy Physics, Beijing, China.

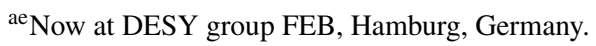

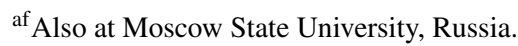

${ }^{\mathrm{ag}}$ Now at University of Liverpool, United Kingdom.

${ }^{\text {ah }}$ Now at CERN, Geneva, Switzerland.

${ }^{\text {ai }}$ Also affiliated with University College London, UK.

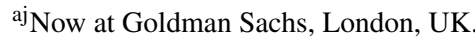

${ }^{\mathrm{ak}}$ Also at Institute of Theoretical and Experimental Physics, Moscow, Russia.

${ }^{\text {al }}$ Also at INP, Cracow, Poland.

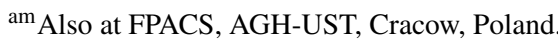

${ }^{a n}$ Partially supported by Warsaw University, Poland.

${ }^{\text {ao }}$ Now at Istituto Nucleare di Fisica Nazionale (INFN), Pisa, Italy.

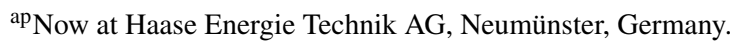

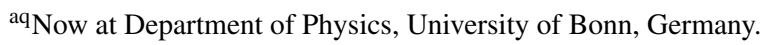

${ }^{a r}$ Now at Biodiversität und Klimaforschungszentrum (BiK-F), Frankfurt, Germany.

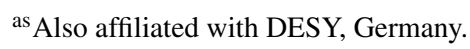

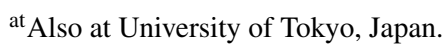

${ }^{\mathrm{au}}$ Now at Kobe University, Japan.

${ }^{\text {av }}$ Supported by DESY, Germany.

${ }^{\text {aw }}$ Member of National Technical University of Ukraine, Kyiv Polytechnic Institute, Kyiv, Ukraine.

${ }^{a x}$ Member of National University of Kyiv-Mohyla Academy, Kyiv, Ukraine.

${ }^{\text {ay }}$ Supported by the Bogolyubov Institute for Theoretical Physics of the National Academy of Sciences, Ukraine.

${ }^{\mathrm{az}}$ STFC Advanced Fellow.

ba Nee Korcsak-Gorzo.

${ }^{\mathrm{bb}}$ This material was based on work supported by the National Science Foundation, while working at the Foundation.

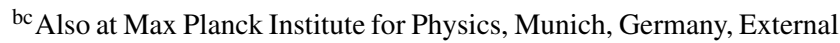
Scientific Member.

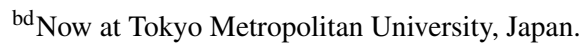

be Now at Nihon Institute of Medical Science, Japan.

bf Now at Osaka University, Osaka, Japan.

bg Also at Łódź University, Poland.

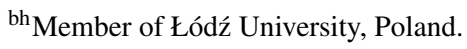

${ }^{b i}$ Now at Lund University, Lund, Sweden.

bj Also at University of Podlasie, Siedlce, Poland.
} 
modynamics (QCD) since the heavy-quark masses provide a hard scale that allows perturbative calculations. At leading order, boson-gluon fusion (BGF), $\gamma g \rightarrow q \bar{q}$ with $q \in$ $\{b, c\}$, is the dominant process for heavy-quark production at HERA. When the negative squared four-momentum exchanged at the electron vertex, $Q^{2}$, is small, the process can be treated as photoproduction, in which a quasi-real photon emitted by the incoming electron interacts with the proton. For heavy-quark transverse momenta larger than or comparable to the quark mass, next-to-leading-order (NLO) QCD calculations in which the massive quarks are generated in the hard sub-process $[1,2]$ are expected to provide reliable predictions for the photoproduction cross sections.

Beauty and charm photoproduction has been measured using several different methods by both the ZEUS and H1 collaborations. In most of the previous measurements of beauty photoproduction at HERA, the cross section was determined using semileptonic decays into muons [3-6] or electrons $[7,8]$. In the muon analyses, the fraction of leptons originating from beauty was determined by using the large transverse momentum of the muon relative to the axis

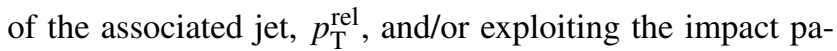
rameter of the muons. In the more recent electron analysis [7], several variables, sensitive to both electron identification as well as to semileptonic decays, were combined in a likelihood-ratio test function in order to extract the beauty and charm content. The $\mathrm{H} 1$ collaboration has published an inclusive measurement of beauty- and charm-quark photoproduction using a method based on the impact parameter of tracks to the primary vertex [9]. The other published charm or beauty photoproduction measurements [10-16] used either meson tags or a combination of lepton and meson tags. In all of the above analyses reasonable agreement between the measurement and the theory prediction was found.

The aim of this measurement is to test perturbative QCD with high precision. For this purpose, the long lifetimes of the weakly decaying $b$ and $c$ hadrons as well as their large masses were exploited. The measurement relies on the reconstruction of decay vertices with the ZEUS silicon microvertex detector (MVD) [17]. Two discriminating variables were used: the significance of the reconstructed decay length and the invariant mass of the charged tracks associated with the decay vertex (secondary vertex). The measurement was kept fully inclusive, leading to a reduced uncertainty due to branching fractions and a substantial increase in statistics compared to exclusive analyses. The high statistics also allowed the kinematic region of the measurement to be extended to high values of the transverse jet momentum, $p_{\mathrm{T}}^{\mathrm{jet}}$.

\section{Experimental set-up}

The analysis was performed with data corresponding to an integrated luminosity of $133 \mathrm{pb}^{-1}$ which were taken during 2005. Electrons at an energy of $E_{\mathrm{e}}=27.5 \mathrm{GeV}$ collided with protons at $E_{\mathrm{p}}=920 \mathrm{GeV}$, yielding a centre-of-mass energy of $318 \mathrm{GeV}$.

A detailed description of the ZEUS detector can be found elsewhere [18]. A brief outline of the components that are most relevant for this analysis is given below.

In the kinematic range of the analysis, charged particles were tracked in the central tracking detector (CTD) [19-21] and the microvertex detector (MVD) [17]. These components operated in a magnetic field of $1.43 \mathrm{~T}$ provided by a thin superconducting solenoid. The CTD consisted of 72 cylindrical drift-chamber layers, organised in nine superlayers covering the polar-angle ${ }^{1}$ region $15^{\circ}<\theta<164^{\circ}$. The MVD silicon tracker consisted of a barrel (BMVD) and a forward (FMVD) section. The BMVD contained three layers and provided polar-angle coverage for tracks from $30^{\circ}$ to $150^{\circ}$. The four-layer FMVD extended the polar-angle coverage in the forward region to $7^{\circ}$. After alignment, the single-hit resolution of the MVD was $24 \mu \mathrm{m}$. The transverse distance of closest approach (DCA) to the nominal vertex in $X-Y$ was measured to have a resolution, averaged over the azimuthal angle, of $\left(46 \oplus 122 / p_{\mathrm{T}}\right) \mu \mathrm{m}$, with $p_{\mathrm{T}}$ in $\mathrm{GeV}$. For CTD-MVD tracks that pass through all nine CTD superlayers, the momentum resolution was $\sigma\left(p_{\mathrm{T}}\right) / p_{\mathrm{T}}=0.0029 p_{\mathrm{T}} \oplus$ $0.0081 \oplus 0.0012 / p_{\mathrm{T}}$, with $p_{\mathrm{T}}$ in $\mathrm{GeV}$.

The high-resolution uranium-scintillator calorimeter (CAL) [22-25] consisted of three parts: the forward (FCAL), the barrel (BCAL) and the rear (RCAL) calorimeters. Each part was subdivided transversely into towers and longitudinally into one electromagnetic section (EMC) and either one (in RCAL) or two (in BCAL and FCAL) hadronic sections (HAC). The smallest subdivision of the calorimeter was called a cell. The CAL energy resolutions, as measured under test-beam conditions, were $\sigma(E) / E=0.18 / \sqrt{E}$ for electrons and $\sigma(E) / E=0.35 / \sqrt{E}$ for hadrons, with $E$ in $\mathrm{GeV}$.

The luminosity was measured using the Bethe-Heitler reaction $e p \rightarrow e \gamma p$ by a luminosity detector which consisted of independent lead-scintillator calorimeter [26-28] and magnetic spectrometer [29] systems. The fractional systematic uncertainty on the measured luminosity was $1.8 \%$.

\footnotetext{
${ }^{1}$ The ZEUS coordinate system is a right-handed Cartesian system, with the $Z$ axis pointing in the nominal proton beam direction, referred to as the "forward direction", and the $X$ axis pointing left towards the centre of HERA. The coordinate origin is at the centre of the CTD. The pseudorapidity is defined as $\eta=-\ln \left(\tan \frac{\theta}{2}\right)$, where the polar angle, $\theta$, is measured with respect to the proton beam direction. The azimuthal angle, $\phi$, is measured with respect to the $X$ axis.
} 


\section{Monte Carlo simulation}

Monte Carlo (MC) samples of beauty, charm and lightflavour events generated with PүтнIA 6.2 [30-32] were used to evaluate the detector acceptance and to provide the predictions of the signal and background distributions.

The production of $b \bar{b}$ and $c \bar{c}$ pairs was simulated following the standard PYTHIA prescription, using leading-order matrix elements combined with parton showering. The following subprocesses [33] were generated:

- Direct and resolved photoproduction with leading-order massive matrix elements. In the direct-photon process, the quasi-real photon enters directly in the hard interaction, while in the resolved-photon process, the photon acts as a source of light partons which take part in the hard interaction. The $b$-quark and $c$-quark masses were set to $4.75 \mathrm{GeV}$ and $1.5 \mathrm{GeV}$, respectively.

- $b$-quark and $c$-quark excitation, i.e. the contribution to the leading-order massless matrix elements of $b$ and $c$ quarks from initial-state photon or gluon splitting.

The light-quark predictions were taken from a simulation of both direct and non-direct inclusive photoproduction with leading-order matrix elements in the massless scheme. This sample also includes final-state gluon splitting into $b \bar{b}$ and $c \bar{c}$ pairs, which is treated as part of the signal.

The CTEQ4L [34] and CTEQ5L [35] proton parton distribution functions (PDFs) were used for the lightflavour and heavy-flavour samples, respectively. The GRV-G LO $[36,37]$ photon PDF was used for all samples.

The lifetimes of the $B^{ \pm}, B^{0}$ and $B_{s}$ mesons were corrected from the default PYTHIA values to reflect the world averages [38].

The generated events were passed through a full simulation of the ZEUS detector based on GEANT 3.21 [39]. The final $\mathrm{MC}$ events had to fulfil the same trigger requirements and pass the same reconstruction programme as the data.

\section{Data selection and event reconstruction}

A three-level trigger system was used to select events online $[18,40,41]$. At the third level, jets were reconstructed using the energies and positions in the CAL. Events with at least two jets with transverse momentum in excess of $4.5 \mathrm{GeV}$ within $|\eta|<2.5$ were selected.

The tracking efficiency at the first-level trigger (FLT) as well as the efficiency of the dijet third-level trigger (TLT) were lowered in the detector simulation such that they reproduced the efficiencies as measured in the data. The trigger efficiencies were $\approx 86 \%$ for the FLT and $76-100 \%$ for the TLT, depending on the transverse momentum of the jets, with an average of about $90 \%$. The average corrections amounted to $\approx 7.7 \%$ for the FLT and $\approx 3.7 \%$ for the TLT.
The hadronic system was reconstructed from energyflow objects (EFOs) [42] combining track and calorimeter information, corrected for energy loss in the dead material. Each $\mathrm{EFO}, i$, was assigned a reconstructed fourmomentum $\left(p_{X}^{i}, p_{Y}^{i}, p_{Z}^{i}, E^{i}\right)$, assuming the pion mass. Jets were reconstructed from EFOs using a $k_{\mathrm{T}}$ clustering algorithm [43] in the longitudinally invariant mode [44]. The $E$ recombination scheme, which produces massive jets whose four-momenta are the sum of the four-momenta of the clustered objects, was used.

At least two jets with $\left|\eta^{\text {jet }}\right|<2.5$ and $p_{\mathrm{T}}^{\text {jet }}>7(6) \mathrm{GeV}$ for the highest (second highest) energetic jet were required. Only events with a well reconstructed primary vertex with $\left|Z_{\mathrm{vtx}}\right|<30 \mathrm{~cm}$ were selected.

In order to remove background from deep inelastic scattering (DIS), events were rejected in which a scatteredelectron candidate was found in the calorimeter with energy $E_{\mathrm{e}}^{\prime}>5 \mathrm{GeV}$ and $y_{\mathrm{e}}<0.9$, with $y_{\mathrm{e}}=1-\frac{E_{\mathrm{e}}^{\prime}}{2 E_{\mathrm{e}}}\left(1-\cos \theta_{\mathrm{e}}^{\prime}\right)$, where $\theta_{\mathrm{e}}^{\prime}$ is the polar angle of the outgoing electron. The event inelasticity, $y$, was reconstructed from the hadronic final state using the Jacquet-Blondel method [45] with $y_{\mathrm{JB}}=$ $\sum_{i}\left(E^{i}-p_{Z}^{i}\right) / 2 E_{\mathrm{e}}$, where the sum runs over all the EFOs. A cut $0.2<y_{\mathrm{JB}}<0.8$ was used to remove residual DIS events and non-ep interactions. These requirements correspond to an effective cut of $Q^{2} \lesssim 1 \mathrm{GeV}^{2}$ with a median of $Q^{2} \approx 10^{-3} \mathrm{GeV}^{2}$, as estimated from simulations.

In order to reconstruct secondary vertices related to $b$ and $c$-hadron decays, tracks were selected if:

- $p_{\mathrm{T}}>0.5 \mathrm{GeV}$.

- The number of superlayers in the CTD $\geq 3$.

- The total number of hits ${ }^{2}$ in the MVD $\geq 4$.

The tracks were associated with one of the two highest energetic jets if they fulfilled

$$
\Delta R=\sqrt{\left(\eta^{\mathrm{trk}}-\eta^{\mathrm{jet}}\right)^{2}+\left(\phi^{\mathrm{trk}}-\phi^{\mathrm{jet}}\right)^{2}}<1 .
$$

If two or more of such tracks were associated with the selected jet, a candidate vertex was fitted from the selected tracks using a deterministic annealing filter [46-48]. This fit provided the vertex position including its error matrix as well as the invariant mass, $m_{\mathrm{vtx}}$, of the charged tracks associated with the reconstructed vertex. Vertices with $\chi^{2} / \mathrm{ndf}<$ 6 , a distance from the interaction point within $1 \mathrm{~cm}$ in the $X$ $Y$ plane and $\pm 30 \mathrm{~cm}$ in the $Z$ direction, and $0.8 \leq m_{\mathrm{vtx}}<$ $7.5 \mathrm{GeV}$ were retained for further analysis.

Only those secondary vertices that were associated with one of the two jets with the highest $p_{\mathrm{T}}^{\text {jet }}$ were considered, since these jets were most likely to correspond to heavyquark jets. The associated jet was required to be reconstructed within the central part of the detector with $-1.6 \leq$ $\eta^{\mathrm{jet}}<1.4$.

${ }^{2}$ Each MVD layer provided two coordinate measurements. 
Fig. 1 Distributions of decay-length significance, $S$, for a $0.8 \leq m_{\mathrm{vtx}}<1.4 \mathrm{GeV}$, b $1.4 \leq m_{\mathrm{vtx}}<2 \mathrm{GeV}$ and c $2 \leq m_{\mathrm{vtx}}<7.5 \mathrm{GeV}$. The data are compared to the total PYTHIA MC distributions as well as the contributions from the beauty, charm and light-flavour MC subsamples. All samples were normalised according to the scaling factors obtained from the fit (see Sect. 8)

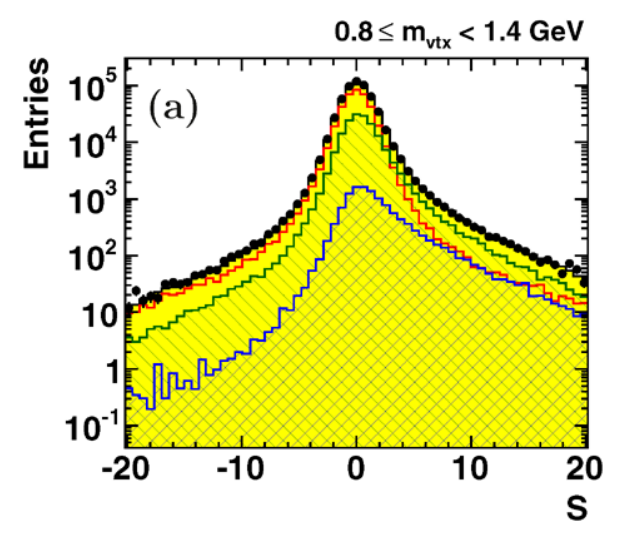

\section{ZEUS}
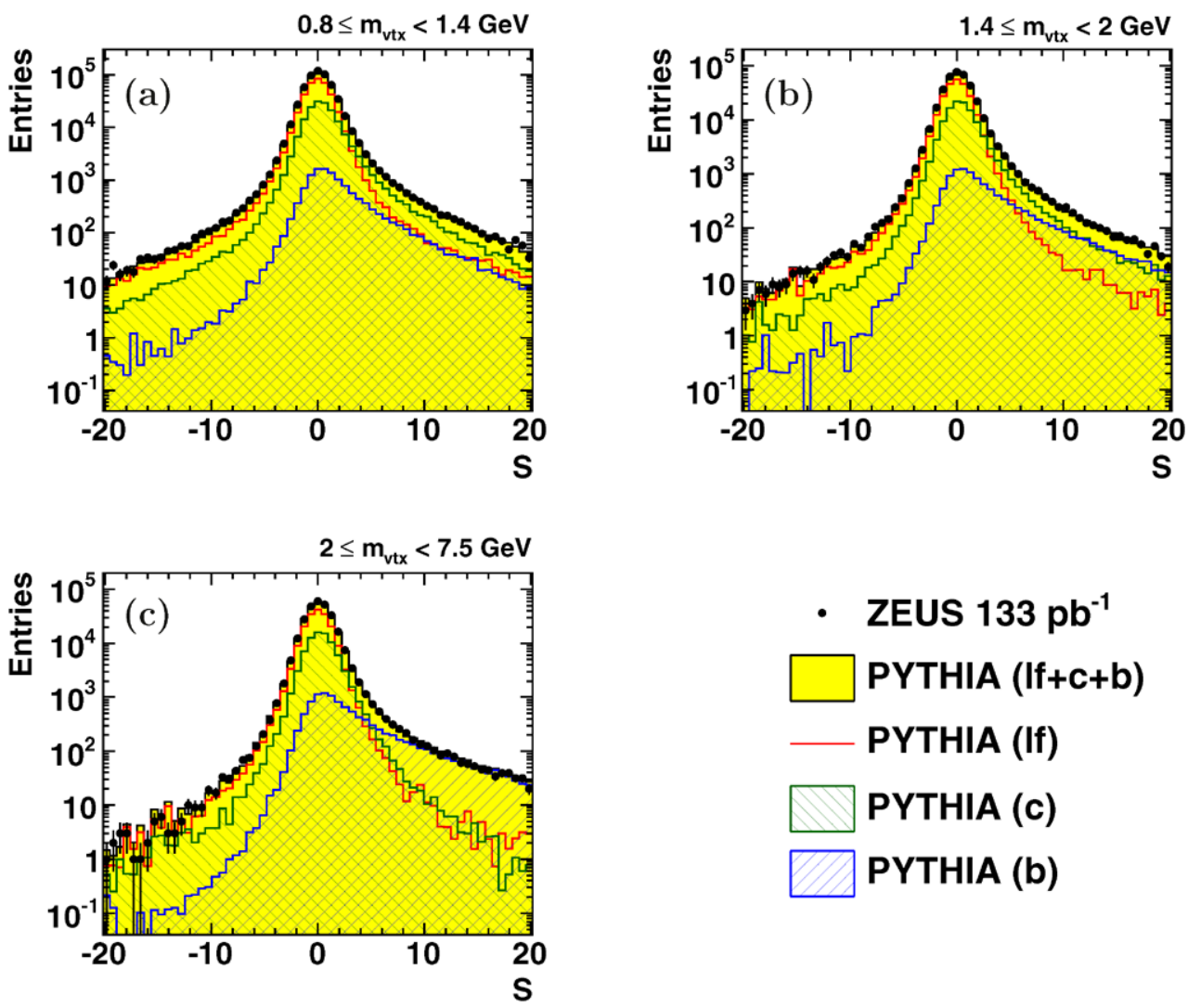

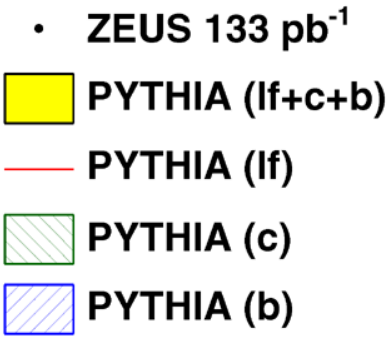

\section{Extraction of the heavy-flavour cross sections}

Using the secondary vertices associated with jets, the decay length, $d$, was defined as the distance in $X-Y$ between the secondary vertex and the interaction point ${ }^{3}$, projected onto the jet axis in the $X-Y$ plane.

The decay-length significance, $S$, was defined as $d / \delta d$, where $\delta d$ is the uncertainty on $d$. The sign of the decay length was assigned using the axis of the jet to which the vertex is associated: if the decay-length vector was in the same hemisphere as the jet axis, a positive sign was assigned to it; otherwise the sign of the decay length was negative. Negative decay lengths, which originate from secondary vertices reconstructed on the wrong side of the interaction point with respect to the direction of the associated jets, are unphysical and caused by detector resolution effects. A small correction [33] to the MC decay-length distribution was applied in order to reproduce the negative decay-length data: $5 \%$ of the tracks in the central region were smeared and an ad-

\footnotetext{
${ }^{3}$ In the $X-Y$ plane, the interaction point was defined as the centre of the beam ellipse, determined using the average primary vertex position for groups of a few thousand events, taking into account the difference in angle between the beam direction and the $Z$ direction. The $Z$ coordinate was taken as the $Z$ position of the primary vertex of the event.
}

ditional smearing was applied to tracks in the tails of the decay-length distribution.

The shape of the decay-length significance distribution together with the secondary-vertex mass distribution, $m_{\mathrm{vtx}}$, is used to extract the beauty and charm content. The invariant mass of the tracks fitted to the secondary vertex provides a distinguishing variable for jets from $b$ and $c$ quarks, reflecting the different masses of the $b$ and $c$ hadrons. Figure 1 shows the decay-length significance, $S$, divided into the three mass bins $0.8 \leq m_{\mathrm{vtx}}<1.4 \mathrm{GeV}, 1.4 \leq m_{\mathrm{vtx}}<2 \mathrm{GeV}$ and $2 \leq m_{\mathrm{vtx}}<7.5 \mathrm{GeV}$. The MC simulation provides a good description of the data in all three bins and an almost pure beauty region can be obtained at high significances in the bin $2 \leq m_{\mathrm{vtx}}<7.5 \mathrm{GeV}$.

In order to minimise the effect of the light-flavour contribution, the contents of the negative bins of the significance distribution, $N\left(S^{-}\right)$, were subtracted from the contents of the corresponding positive bins, $N\left(S^{+}\right)$, yielding a subtracted decay-length significance distribution. An additional advantage of this subtraction is that symmetric systematic effects, which might arise from discrepancies between the data and the MC, are removed.

In order further to reduce the uncertainty due to remaining differences between data and $\mathrm{MC}$ in the core region of the significance distribution, a cut of $|S|>3$ was applied. 
Fig. 2 Distributions of a $\eta^{\text {jet }}$ and $\mathbf{b} p_{\mathrm{T}}^{\text {jet }}$ of the jets associated with a secondary vertex, $\mathbf{c} m_{\mathrm{vtx}}$ and $\mathbf{d} n_{\text {trk }}$ of the selected secondary vertices. e $\chi^{2} /$ ndf of the secondary vertices before the cut shown in the figure had been applied. f shows $x_{\gamma}^{\text {jet }}$ weighted by the number of jets with associated secondary vertices in the event. The data are compared to the total MC distributions as well as the contributions from the beauty and charm MC subsamples. All samples were normalised according to the scaling factors obtained from the fit (see Sect. 8)

Fig. 3 Distribution of the subtracted decay-length significance in three mass bins. The data are compared to the total PYTHIA MC distribution as well as the contributions from the beauty, charm and light-flavour MC subsamples. All samples were normalised according to the scaling factors obtained from the fit

\section{ZEUS}
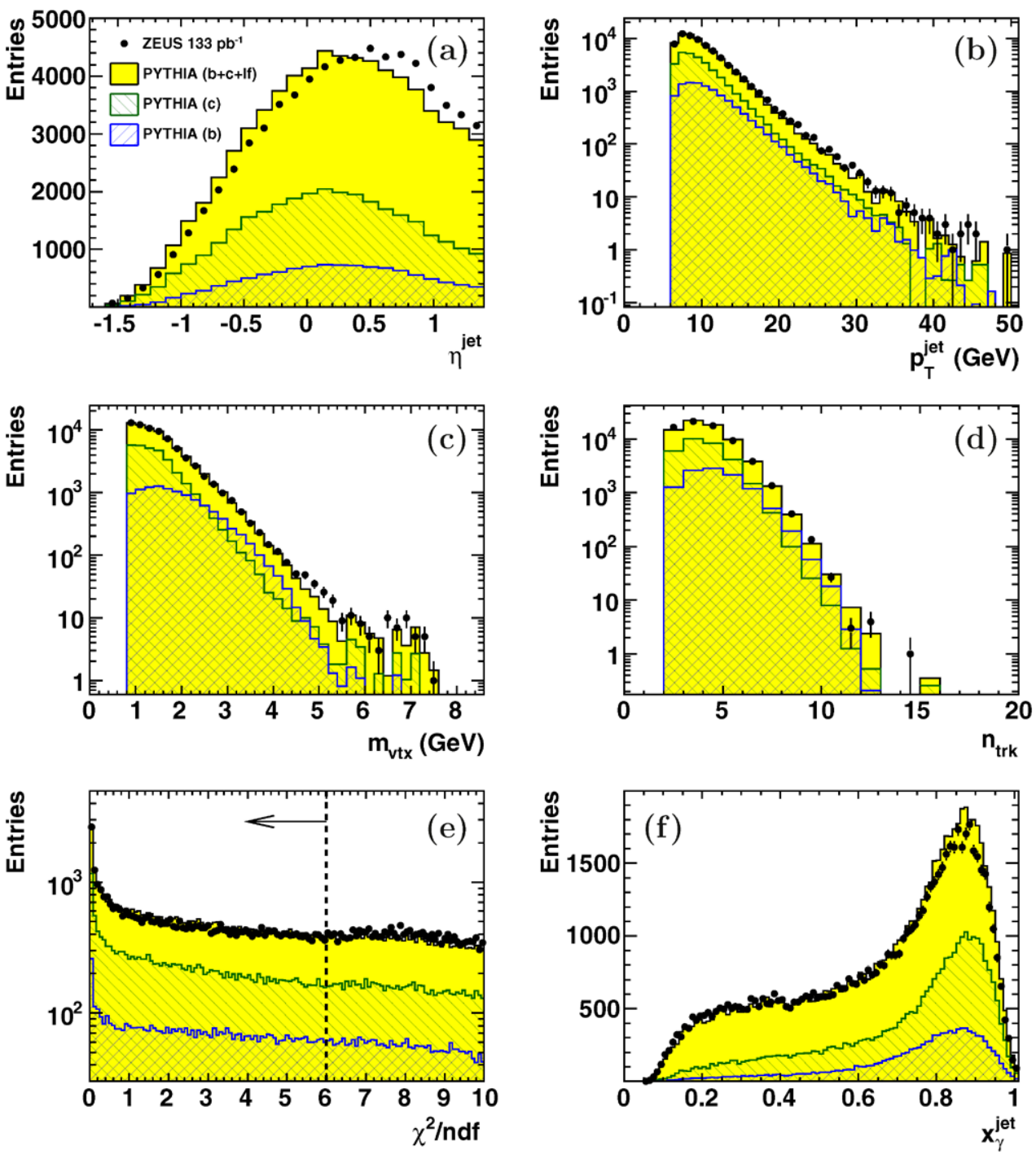

\section{ZEUS}

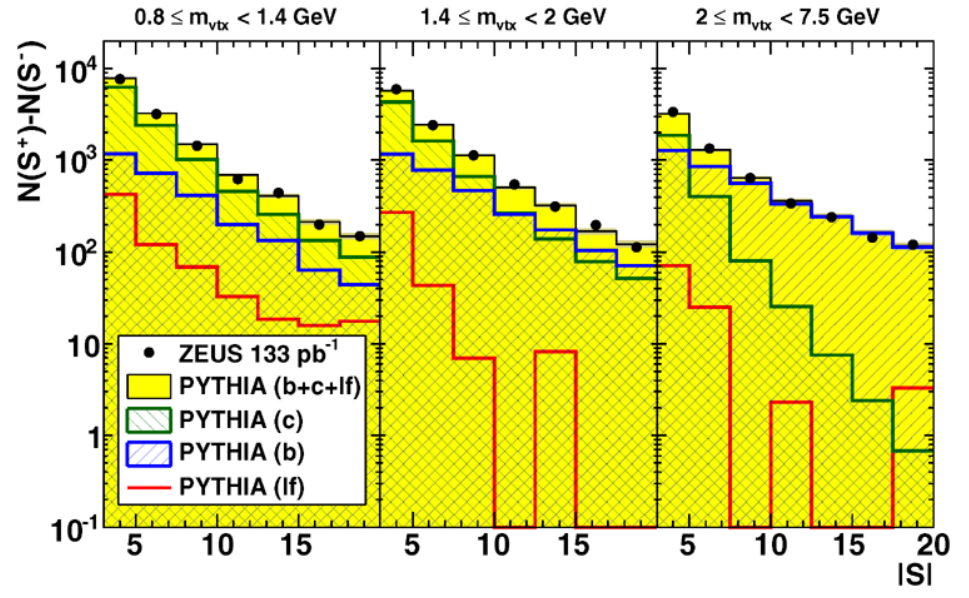




\section{ZEUS}
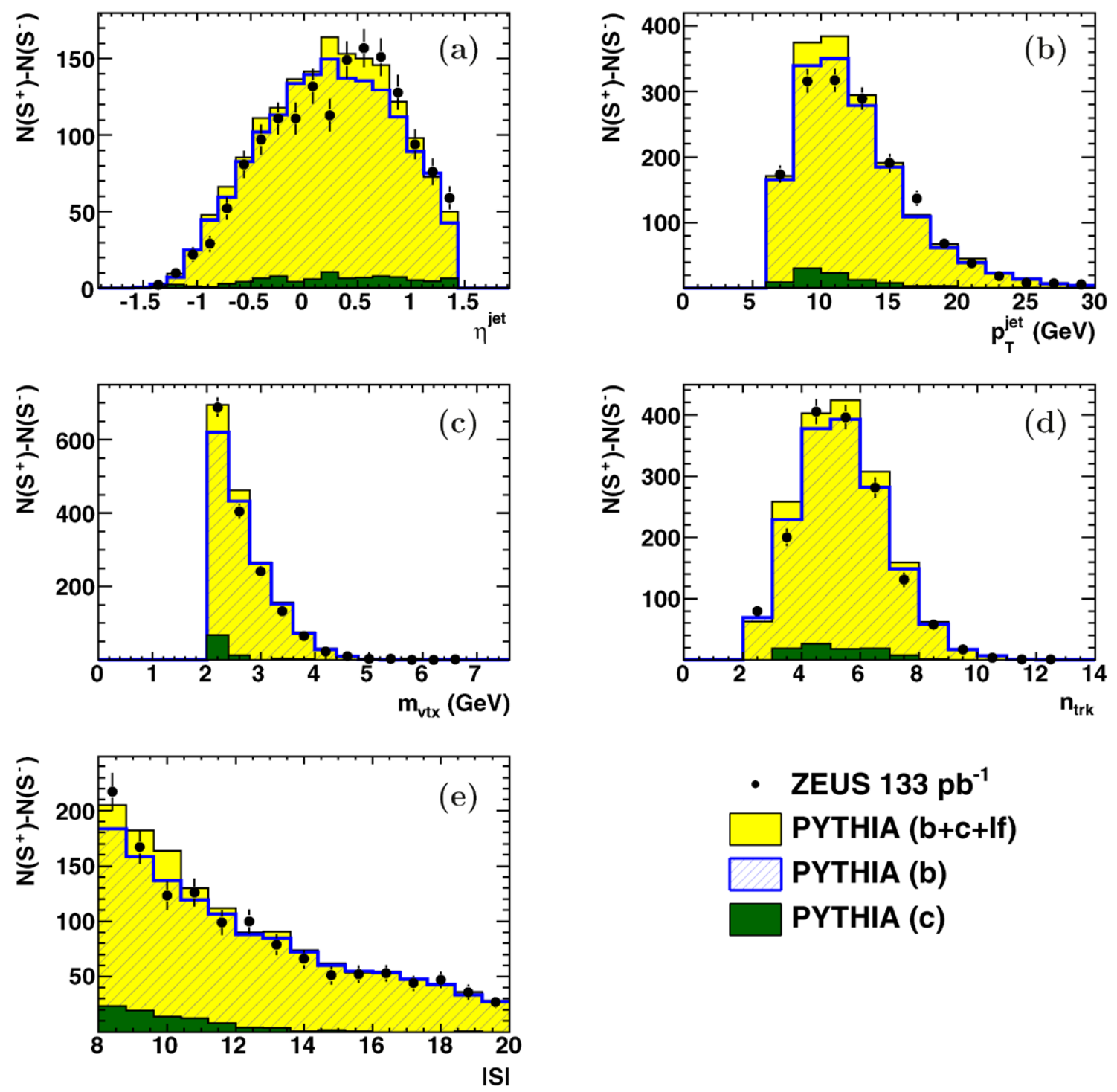

Fig. 4 Distributions of $\mathbf{a} \eta^{\text {jet }}, \mathbf{b} p_{\mathrm{T}}^{\text {jet }}, \mathbf{c} m_{\mathrm{vtx}}$ and $\mathbf{d} n_{\text {trk }}$ of the selected secondary vertices and e subtracted decay-length significance, for a beauty-enriched subsample with $2 \leq m_{\mathrm{vtx}}<7.5 \mathrm{GeV}$ and $|S|>8$. The data are compared to the total MC distributions as well as the contri-

butions from the beauty and charm MC subsamples. The light-flavour contribution is not shown separately as it is negligible on the scales shown. All samples were normalised according to the scaling factors obtained from the fit

As a consistency check this cut was varied in order to estimate the uncertainty due to the MC modelling of the low $|S|$ region; effects smaller than $1 \%$ on the beauty results and $3 \%$ on the charm results were found.

After all selection cuts, a sample of 70433 jets with associated secondary vertices remained.

Figure 2 shows the data and MC distributions of $p_{\mathrm{T}}^{\text {jet }}$, $\eta^{\text {jet }}, m_{\mathrm{vtx}}$, the secondary vertex track multiplicity, $n_{\text {trk }}$, and $\chi^{2} /$ ndf of the secondary vertices. All distributions are shown after all selection cuts, except for the $\chi^{2} /$ ndf distribution, where the $\chi^{2} /$ ndf cut has not been applied yet. Also

shown in Fig. 2 is the fraction of the total hadronic $E-p_{Z}$ carried by the two highest- $p_{\mathrm{T}}$ jets,

$x_{\gamma}^{\text {jet }}=\frac{\sum_{j=1,2}\left(E^{j}-p_{Z}^{j}\right)}{E-p_{Z}}$,

weighted by the number of jets with associated secondary vertices in the event. This distribution is sensitive to the fraction of direct and non-direct photoproduction contributions. The MC provides an adequate description of the data for all variables except $\eta^{\text {jet}}$; the effect of this discrepancy on the results is discussed in Sect. 6. 


\section{ZEUS}
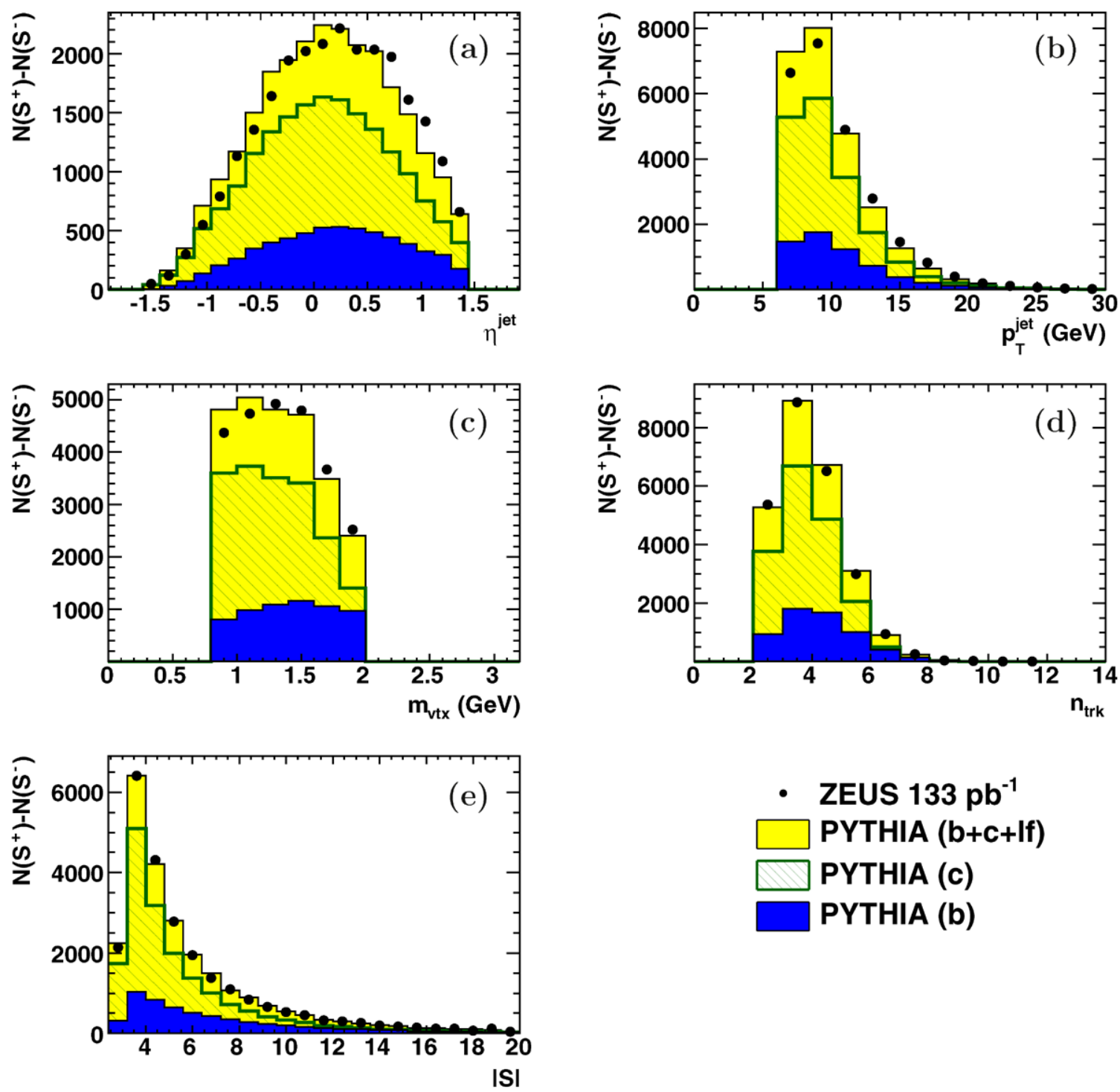

Fig. 5 Distributions of $\mathbf{a} \eta^{\text {jet }}, \mathbf{b} p_{\mathrm{T}}^{\text {jet }}, \mathbf{c} m_{\mathrm{vtx}}$ and $\mathbf{d} n_{\text {trk }}$ of the selected secondary vertices and e subtracted decay-length significance, for a charm-enriched subsample with $0.8 \leq m_{\mathrm{vtx}}<2 \mathrm{GeV}$. No additional significance cut was applied here. The data are compared to the to-

The beauty and charm contributions were extracted using a least-squares fit $[33,49]$ to the subtracted distributions in the three mass bins. The MC beauty, charm and light-flavour contributions, normalised to the data luminosity, were scaled by the factors $k_{b}, k_{c}$ and $k_{\mathrm{lf}}$, respectively, to give the best fit to the observed subtracted distributions. The overall MC normalisation was constrained by requiring it to be consistent with the normalisation of the data in the significance distribution with $|S|>3$ and $0.8 \leq m_{\mathrm{vtx}}<7.5 \mathrm{GeV}$. The subtracted and fitted distributions for the three mass bins are shown in Fig. 3. The

tal MC distributions as well as the contributions from the beauty and charm MC subsamples. The light-flavour contribution is not shown separately as it is negligible on the scales shown. All samples were normalised according to the scaling factors obtained from the fit

contribution of the light flavours was substantially reduced through the subtraction. After the subtraction, good agreement was also observed between the data and the MC simulation. The fit procedure was repeated in different bins of $p_{\mathrm{T}}^{\text {jet }}$ and $\eta^{\text {jet }}$ to obtain the differential cross-sections $\mathrm{d} \sigma / \mathrm{d} p_{\mathrm{T}}^{\text {jet }}$ and $\mathrm{d} \sigma / \mathrm{d} \eta^{\text {jet }}$.

In order to check the quality of the data description by the MC, subtracted distributions of $p_{\mathrm{T}}^{\text {jet }}, \eta^{\text {jet }}, m_{\mathrm{vtx}}$, the secondary-vertex track multiplicity, $n_{\text {trk }}$, and $|S|$ are shown in Fig. 4 after beauty enrichment $\left(2 \leq m_{\mathrm{vtx}}<7.5 \mathrm{GeV}\right.$ and 
$|S| \geq 8)$ and in Fig. 5 after charm enrichment $\left(0.8 \leq m_{\mathrm{vtx}}<\right.$ $2 \mathrm{GeV})$.

The total visible cross section for inclusive heavy-quark jet production, $\sigma^{q}$, with $q \in\{b, c\}$ is given by

$\sigma^{q}=\frac{N_{q}^{\text {rec,Data }}}{\mathcal{A}_{q} \cdot \mathcal{L}_{\text {Data }}}$.

Here, $\mathcal{L}_{\text {Data }}$ denotes the integrated luminosity, $\mathcal{A}_{q}$ is the acceptance and $N_{q}^{\text {rec,Data }}$ the number of reconstructed heavyquark jets in data, which was determined from the fit using

$N_{q}^{\text {rec,Data }}=k_{q} \cdot N_{q}^{\mathrm{rec}, \mathrm{MC}}$,

with $N_{q}^{\text {rec,MC }}$ being the number of reconstructed events in a MC sample with the same integrated luminosity as the data. $k_{q}$ denotes the heavy-quark scaling factor obtained from the fit. Defining the acceptance as

$\mathcal{A}_{q}=\frac{N_{q}^{\mathrm{rec}, \mathrm{MC}}}{N_{q}^{\text {true, } \mathrm{HL}}}$,

the cross section can be written as

$\sigma^{q}=\frac{k_{q} \cdot N_{q}^{\text {true, } \mathrm{HL}}}{\mathcal{L}^{\text {Data }}}$.

Here, $N_{q}^{\text {true,HL }}$ denotes the number of generated heavyquark jets at hadron level (HL). Hadron-level jets were obtained by running the $k_{\mathrm{T}}$ clustering algorithm in the same mode as for the data with the $E$-recombination scheme. The algorithm was run on all final-state MC particles before the decay of the weakly decaying $b$ or $c$ hadrons. True $b$ or $c$ jets were then defined as all hadron-level jets containing a $b$ or $c$ hadron. Signatures with $b$ or $c$ hadrons resulting from final-state gluon splitting ( $g \rightarrow q \bar{q}$ ) were also included in the respective signal, independent of the quark flavours in the hard subprocess. The contribution of gluon splitting to the beauty signal amounted to $\approx 2 \%$, while the contribution to the charm signal was $\approx 10 \%$.

The single-differential heavy-quark jet production cross section as a function of a given variable, $v$, is defined accordingly:

$\frac{\mathrm{d} \sigma^{q}}{\mathrm{~d} v}=\frac{k_{q} \cdot N_{q}^{\mathrm{true}, \mathrm{HL}}}{\mathcal{L}^{\text {Data }} \cdot \Delta v}$,

where $\Delta v$ is the width of the bin.

\section{Systematic uncertainties}

Systematic uncertainties were evaluated by appropriate variations of the MC simulation. The fit of the subtracted decaylength significance in $m_{\mathrm{vtx}}$ bins was repeated and the cross
Table 1 Systematic uncertainties on the total beauty- and charm-jet cross sections

Source

Beauty/Charm

$(\%)$

\begin{tabular}{|c|c|c|}
\hline (1a) & TLT trigger efficiency & $\pm 0.8 / \pm 2.0$ \\
\hline (1b) & FLT trigger efficiency & ${ }_{-3.8}^{+4.1} /{ }_{-3.7}^{+4.0}$ \\
\hline (2) & CAL hadronic energy scale & $\pm 0.6 / \pm 4.3$ \\
\hline (3) & Track-finding uncertainty & $+5.9 /+1.0$ \\
\hline (4) & Decay-length smearing & $\pm 1.0 / \pm 0.7$ \\
\hline (5) & Light-flavour asymmetry & $\pm 0.2 / \pm 0.7$ \\
\hline (6a) & $\eta^{\text {jet }}$ reweighting & $-1.2 /-1.0$ \\
\hline (6b) & $p_{\mathrm{T}}^{\text {jet }}$ reweighting & $-5.5 /-1.1$ \\
\hline (7a) & $D^{ \pm} / D^{0}$ ratio & ${ }_{-1.3}^{+0} /{ }_{-1.8}^{+0.6}$ \\
\hline (7b) & $D^{ \pm} / D_{s}^{ \pm}$ratio & ${ }_{-1,2}^{-1.5} / /_{-1,3}^{+0.0}$ \\
\hline (8) & Charm fragmentation & ${ }_{-0.3}^{+0.3} / /_{-1.3}^{+1.2}$ \\
\hline (9) & Beauty fragmentation & ${ }_{-2.1}^{+1.8} /{ }_{-0.1}^{+0.1}$ \\
\hline \multirow[t]{2}{*}{ (10) } & Luminosity measurement & $\pm 1.8 / \pm 1.8$ \\
\hline & Total & ${ }_{-7.7}^{+7.8} /{ }_{-7.0}^{+6.7}$ \\
\hline
\end{tabular}

sections were recalculated. The uncertainties on the total cross sections determined for each source are summarised in Table 1. The following sources of experimental systematic uncertainties were identified [33]:

1. The systematic uncertainties associated with the TLT and FLT trigger efficiency corrections (see Sect. 4) were determined by varying each correction within its estimated uncertainty.

2. The calorimetric part of the jet energy was varied by $\pm 3 \%$.

3. The track-finding inefficiency in the data with respect to the MC was estimated to be at most $2 \%$. The overall uncertainty due to this tracking inefficiency was determined by randomly rejecting $2 \%$ of all tracks in the MC and repeating the secondary vertex finding and all subsequent analysis steps;

4. The uncertainty due to the smearing procedure was estimated by varying the fraction of secondary vertices for which the decay length was smeared by $\pm 2 \%$. For variations of the fraction in this range the agreement between data and $\mathrm{MC}$ remained reasonable.

5. The uncertainty due to the asymmetry of the lightflavour content of the sample was evaluated by varying $k_{\text {lf }}$ by $\pm 11 \%$. The size of the variation was estimated from the uncertainty on the light-flavour fraction as determined by a fit to the subtracted decay-length significance distribution, where the overall normalisation constraint using the unsubtracted distribution was not applied.

6. The MC distributions for both light and heavy flavours were reweighted in $\eta^{\text {jet }}$ and $p_{\mathrm{T}}^{\text {jet }}$ to account for the differences between data and MC (see Fig. 2). A reweight- 
ing of only the light-flavour content was also investigated. No significant change of the cross sections was observed and therefore no additional systematic uncertainty was assigned.

7. The various $D$ mesons have different lifetimes and decay modes. In order to account for the uncertainty of the different fragmentation fractions, the $D^{+} / D^{0}$ and $D^{+} / D_{s}^{+}$ratios were varied by $\pm 10 \%$ while keeping the total number of $c$ hadrons constant.

8. The charm fragmentation function was varied by weighting all events according to

$$
z=\frac{\left(E+P_{\| \mid}\right)_{D}}{(E+P)_{c \text {-quark jet }}}
$$

calculated in the string rest-frame [30-32] such that the change in the mean value of $z$ corresponded to the measured uncertainty [50].

9. The beauty fragmentation function was varied in analogy to the charm case using a variation of $z$ corresponding to a variation of the Peterson fragmentation parameter, $\varepsilon_{b}$, of $\pm 0.0015[51,52]$.

10. A $1.8 \%$ overall normalisation uncertainty was associated with the luminosity measurement. It was included in the systematic error on the total cross sections, but not in those of the differential cross sections.

The same variations were applied to each bin for the differential cross sections. The total systematic uncertainty was obtained by adding the above contributions in quadrature. In the case of beauty, the dominant effects arise from the variation of the trigger-efficiency corrections, the trackfinding efficiency and the reweighting as a function of $p_{\mathrm{T}}^{\text {jet }}$. For charm, the variation of the trigger-efficiency corrections as well as the energy-scale variation contribute most to the total systematic uncertainty.

As an additional consistency check, the contributions of direct and non-direct photon processes were investigated by reweighting the $x_{\gamma}^{\text {jet }}$ distributions based on MC and data comparisons of the $b$ - and $c$-enriched samples. The effect on the cross sections was smaller than that due to the reweighting of the $p_{\mathrm{T}}^{\text {jet }}$ and $\eta^{\text {jet }}$ distributions and so a further contribution was not added to the systematic uncertainties. A reweighting of the $m_{\mathrm{vtx}}$ distribution was also done in order to account for residual differences between the data and the MC. Its effect on the cross sections was found to be negligible.

\section{Theoretical predictions and uncertainties}

The measured total and differential cross sections were compared to NLO QCD predictions calculated with the FMNR programme [53]. This calculation is based on the fixedflavour-number scheme, using three light flavours for the charm predictions and four for beauty. The PDFs were taken from CTEQ6.6 [54] for the proton and GRV-G HO [36] for the photon. The heavy-quark masses (pole masses) were set to $m_{b}=4.75 \mathrm{GeV}$ and $m_{c}=1.5 \mathrm{GeV}$. The QCD scale, $\Lambda_{\mathrm{QCD}}^{(5)}$, was set to $0.226 \mathrm{GeV}$. The renormalisation scale, $\mu_{R}$, and the factorisation scale, $\mu_{F}$, were chosen to be equal and set to $\mu_{R}=\mu_{F}=\frac{1}{2} \sqrt{\hat{p}_{\mathrm{T}}^{2}+m_{b(c)}^{2}}$, where $\hat{p}_{\mathrm{T}}$ is the average transverse momentum of the heavy quarks. In order to ease the comparison with previous analyses, the theoretical predictions were also made using the CTEQ5M [35] proton PDFs.

For the systematic uncertainty on the theoretical prediction, the masses and scales were varied separately and the effects of both variations were added in quadrature. The masses were varied using the values $m_{b}=4.5$ and $5.0 \mathrm{GeV}$, $m_{c}=1.3$ and $1.7 \mathrm{GeV}$; the scales were varied using $\mu_{R}=$ $\mu_{F}=\frac{1}{4} \sqrt{\hat{p}_{\mathrm{T}}^{2}+m_{b(c)}^{2}}$ and $\sqrt{\hat{p}_{\mathrm{T}}^{2}+m_{b(c)}^{2}}$. The resulting uncertainties on the NLO QCD predictions for the total cross sections are $+22 \%$ and $-15 \%$ for beauty and $+42 \%$ and $-21 \%$ for charm.

Parton-level jets were found by applying the $k_{\mathrm{T}}$ clustering algorithm to the generated partonic final state in the same mode as for the hadron level in the MC (see Sect. 5). The NLO QCD predictions for parton-level jets were corrected for hadronisation effects. A bin-by-bin procedure was used whereby $\mathrm{d} \sigma=\mathrm{d} \sigma_{\mathrm{NLO}} \cdot C_{\mathrm{had}}$, and $\mathrm{d} \sigma_{\mathrm{NLO}}$ is the cross section for partons in the final state of the NLO calculation. The hadronisation-correction factors, $C_{\text {had }}$, were obtained from the ratio of the hadron-level to the parton-level MC jet cross section, where the parton level is defined as being the result of the parton-showering stage of the simulation. The correction factors are given in Tables 2 and 3; their uncertainty was negligible in comparison to the other theoretical uncertainties [3].

\section{Results}

The total and single-differential beauty- and charm-jet cross sections were measured for the processes

$e^{-} p \rightarrow e^{-} b(\bar{b}) X$,

$e^{-} p \rightarrow e^{-} c(\bar{c}) X$

in events with

$$
Q^{2}<1 \mathrm{GeV}^{2}, \quad 0.2<y<0.8,
$$$$
p_{\mathrm{T}}^{\text {jet } 1(2)}>7(6) \mathrm{GeV}, \quad-2.5<\eta^{\text {jet } 1(2)}<2.5 \text {. }
$$ 
Here $\eta^{\text {jet 1(2) }}$ and $p_{\mathrm{T}}^{\text {jet 1(2) }}$ refer, respectively, to the pseudorapidities and the transverse momenta of the two jets in the event with the largest transverse momentum within the range $\left|\eta j^{\text {jet }}\right|<2.5$. The cross sections are measured for those jets among these two satisfying

$-1.6<\eta^{q-\text { jet }}<1.4$

with $q \in\{b, c\}$.

The total beauty- and charm-jet production cross sections were measured as

$\sigma_{b}^{\text {vis }}=682 \pm 21$ (stat.) $)_{-52}^{+52}$ (syst.) pb,

$\sigma_{c}^{\text {vis }}=5780 \pm 120$ (stat.) ${ }_{-410}^{+390}$ (syst.) pb.

The errors given correspond to the statistical uncertainties and the total systematic uncertainties including the errors due to the uncertainty in the luminosity measurement. The measurements were compared to NLO QCD predictions calculated with the FMNR programme using the specifications given in Sect. 7:

$\sigma_{b}^{\mathrm{NLO}} \otimes C_{\mathrm{had}}^{b}=740_{-130}^{+210} \mathrm{pb}$,

$\sigma_{c}^{\mathrm{NLO}} \otimes C_{\mathrm{had}}^{c}=6000_{-1300}^{+2400} \mathrm{pb}$.

Hadronisation corrections of $C_{\text {had }}^{b}=0.84$ and $C_{\text {had }}^{c}=0.83$ were applied to the NLO QCD predictions. Good agreement between the measured cross sections and the NLO QCD predictions is observed. Replacing CTEQ6.6 by CTEQ5M as proton PDF reduces the theory predictions by $\approx 5 \%$.
The beauty and charm cross sections as a function of $p_{\mathrm{T}}^{\text {jet }}$ and $\eta^{\text {jet }}$ are given in Tables 2 and 3, respectively, and are shown in Fig. 6. The measurements are compared to the NLO QCD predictions and to the PYTHIA MC scaled (see Sect. 5) by a factor of 1.11 for beauty and 1.35 for charm, as obtained from the inclusive fit. The NLO QCD predictions are in good agreement with the data and the scaled PYTHIA MC describes the distributions well.

In Fig. 7 the $b$-jet cross section, $\mathrm{d} \sigma / \mathrm{d} \eta^{\text {jet }}$, is compared to a previously published analysis [55] using semileptonic decays into muons in dijet events. Both measurements agree well. The improved precision of this analysis can be clearly seen. While a direct comparison with a previous $\mathrm{H} 1$ measurement using a similar approach [9] is not possible, as the cross-section definitions are different, the relative errors on the measurements in this paper are approximately a factor 3 (2) smaller for beauty (charm).

In order to enable direct comparisons with other ZEUS measurements given at the $b$-quark level [3-5, 7, 8, 13], the NLO QCD prediction corrected for hadronisation was used to extrapolate the dijet cross sections to inclusive $b$-quark cross sections:

$\frac{\mathrm{d} \sigma}{\mathrm{d} p_{\mathrm{T}}^{b}}=\frac{\left(\frac{\mathrm{d} \sigma}{\mathrm{d} p_{\mathrm{T}}^{\text {jet }}}\right)^{\mathrm{vis}}}{\left(\frac{\mathrm{d} \sigma}{\mathrm{d} p_{\mathrm{T}}^{\text {jet }}}\right)^{\mathrm{NLO}}} \cdot\left(\frac{\mathrm{d} \sigma}{\mathrm{d} p_{\mathrm{T}}^{b}}\right)^{\mathrm{NLO}}$.

For the previous measurements, the extrapolations have been updated using the CTEQ6.6 proton PDFs. In Fig. 8, the
Table 2 Summary table of differential beauty-jet photoproduction cross sections, as defined in Sect. 8. The measurements are given together with their statistical and systematic uncertainties. The NLO QCD predictions using CTEQ6.6 and their uncertainty are also listed. The last column gives the hadronisation correction factors, $C_{\text {had }}^{b}$

\begin{tabular}{|c|c|c|c|}
\hline $\begin{array}{l}p_{\mathrm{T}}^{\text {jet }} \\
(\mathrm{GeV})\end{array}$ & $\begin{array}{l}\mathrm{d} \sigma_{b} / \mathrm{d} p_{\mathrm{T}}^{\text {jet }} \\
(\mathrm{pb} / \mathrm{GeV})\end{array}$ & $\begin{array}{l}\mathrm{d} \sigma_{b}^{\mathrm{NLO}} / \mathrm{d} p_{\mathrm{T}}^{\mathrm{jet}} \otimes C_{\mathrm{had}}^{b} \\
(\mathrm{pb} / \mathrm{GeV})\end{array}$ & $C_{\text {had }}^{b}$ \\
\hline $6: 11$ & $95.6 \pm 4.9_{-7.0}^{+9.8}$ & $109_{-19}^{+31}$ & 0.83 \\
\hline $11: 16$ & $24.8 \pm 1.2_{-1.4}^{+1.8}$ & $29.1_{-4.7}^{+7.9}$ & 0.89 \\
\hline $16: 21$ & $6.02 \pm 0.49_{-0.57}^{+0.55}$ & $7.1_{-1.2}^{+2.0}$ & 0.92 \\
\hline $21: 27$ & $0.93 \pm 0.22_{-0.20}^{+0.31}$ & $1.87_{-0.34}^{+0.54}$ & 0.95 \\
\hline $27: 35$ & $0.30 \pm 0.12_{-0.12}^{+0.14}$ & $0.46_{-0.08}^{+0.13}$ & 1.05 \\
\hline \multirow[t]{2}{*}{$\eta^{\text {jet }}$} & $\mathrm{d} \sigma_{b} / \mathrm{d} \eta^{\mathrm{jet}}$ & $\mathrm{d} \sigma_{b}^{\mathrm{NLO}} / \mathrm{d} \eta^{\mathrm{jet}} \otimes C_{\mathrm{had}}^{b}$ & $C_{\mathrm{had}}^{b}$ \\
\hline & (pb) & $(\mathrm{pb})$ & \\
\hline$-1.6:-1.1$ & $57 \pm 22_{-3}^{+13}$ & $72_{-13}^{+22}$ & 0.70 \\
\hline$-1.1:-0.8$ & $121 \pm 21_{-16}^{+16}$ & $182_{-30}^{+50}$ & 0.78 \\
\hline$-0.8:-0.5$ & $214 \pm 22_{-12}^{+22}$ & $255_{-42}^{+69}$ & 0.79 \\
\hline$-0.5:-0.2$ & $233 \pm 21_{-21}^{+28}$ & $307_{-50}^{+83}$ & 0.79 \\
\hline$-0.2: 0.1$ & $264 \pm 22_{-23}^{+28}$ & $342_{-55}^{+91}$ & 0.81 \\
\hline $0.1: 0.5$ & $316 \pm 21_{-17}^{+23}$ & $346_{-57}^{+96}$ & 0.86 \\
\hline $0.5: 1.4$ & $288 \pm 15_{-30}^{+20}$ & $265_{-48}^{+82}$ & 0.93 \\
\hline
\end{tabular}


Table 3 Summary table of differential charm-jet photoproduction cross sections, as defined in Sect. 8. The measurements are given systematic uncertainties. The NLO QCD predictions using CTEQ6.6 and their uncertainty are also listed. The last column gives the hadronisation correction factors, $C_{\mathrm{had}}^{c}$ together with their statistical and

\begin{tabular}{llll}
\hline$p_{\mathrm{T}}^{\text {jet }}$ & $\mathrm{d} \sigma_{c} / \mathrm{d} p_{\mathrm{T}}^{\mathrm{jet}}$ & $\mathrm{d} \sigma_{c}^{\mathrm{NLO}} / \mathrm{d} p_{\mathrm{T}}^{\mathrm{jet}} \otimes C_{\mathrm{had}}^{c}$ & $C_{\mathrm{had}}^{c}$ \\
$(\mathrm{GeV})$ & $(\mathrm{pb} / \mathrm{GeV})$ & $(\mathrm{pb} / \mathrm{GeV})$ & \\
\hline
\end{tabular}

\begin{tabular}{rccr}
\hline $6: 11$ & $906 \pm 24_{-60}^{+56}$ & $967_{-210}^{+380}$ & 0.82 \\
$11: 16$ & $194 \pm 7_{-20}^{+20}$ & $192_{-41}^{+75}$ & 0.90 \\
$16: 21$ & $39.1 \pm 3.3_{-6.4}^{+6.4}$ & $38.5_{-8.5}^{+15}$ & 0.92 \\
$21: 27$ & $10.5 \pm 2.1_{-4.0}^{+4.4}$ & $8.9_{-2.0}^{+3.4}$ & 0.90 \\
$27: 35$ & $0.9 \pm 0.7_{-0.9}^{+0.4}$ & $1.96_{-0.43}^{+0.72}$ & 0.91 \\
\hline$\eta^{\text {jet }}$ & $\mathrm{d} \sigma_{c} / \mathrm{d} \eta^{\text {jet }}$ & $\mathrm{d} \sigma_{c}^{\mathrm{NLO}} / \mathrm{d} \eta^{\text {jet }} \otimes C_{\text {had }}^{c}$ & $C_{\text {had }}^{c}$
\end{tabular}

(pb)

(pb)

\begin{tabular}{lccr}
\hline$-1.6:-1.1$ & $499 \pm 79_{-46}^{+36}$ & $825_{-180}^{+320}$ & 0.71 \\
$-1.1:-0.8$ & $1380 \pm 110_{-110}^{+110}$ & $1933_{-400}^{+700}$ & 0.79 \\
$-0.8:-0.5$ & $2090 \pm 120_{-180}^{+140}$ & $2566_{-540}^{+940}$ & 0.80 \\
$-0.5:-0.2$ & $2460 \pm 130_{-170}^{+170}$ & $2948_{-610}^{+1100}$ & 0.80 \\
$-0.2: 0.1$ & $2920 \pm 130_{-220}^{+200}$ & $2975_{-630}^{+1100}$ & 0.83 \\
$0.1: 0.5$ & $2600 \pm 110_{-260}^{+180}$ & $2602_{-560}^{+1000}$ & 0.87 \\
$0.5: 1.4$ & $2040 \pm 91_{-140}^{+160}$ & $1579_{-360}^{+700}$ & 0.89 \\
\hline
\end{tabular}

\section{ZEUS}
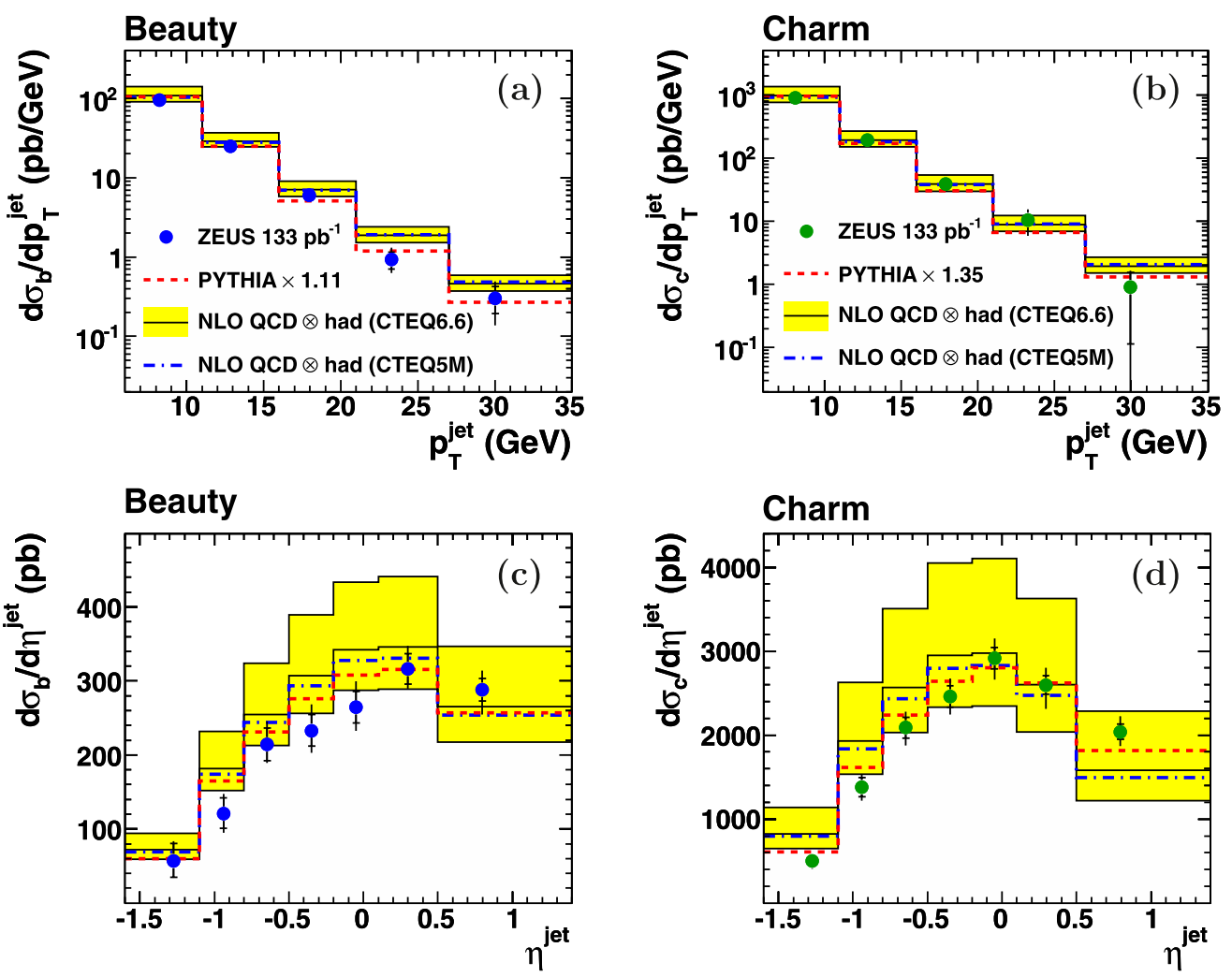

Fig. 6 Differential beauty-jet and charm-jet photoproduction cross sections as defined in Sect. 8 as a function of $\mathbf{a}-\mathbf{b} p_{\mathrm{T}}^{\text {jet }}$ and $\mathbf{c}-\mathbf{d} \eta^{\text {jet }}$. The data are shown as points. The inner error bars are the statistical uncertainties, while the outer error bars show the statistical and systematic uncertainties added in quadrature. The band represents the NLO QCD prediction, corrected for hadronisation effects, using CTEQ6.6 as proton PDF; the shaded band shows the estimated uncertainty. The NLO QCD prediction using CTEQ5M as proton PDF is depicted separately (dotted-dashed line). The scaled PYTHIA MC prediction (dashed line) is also shown 
$b$-quark differential cross sections as a function of the quark transverse momentum, $\mathrm{d} \sigma(e p \rightarrow b X) / \mathrm{d} p_{\mathrm{T}}^{b}$, are shown for

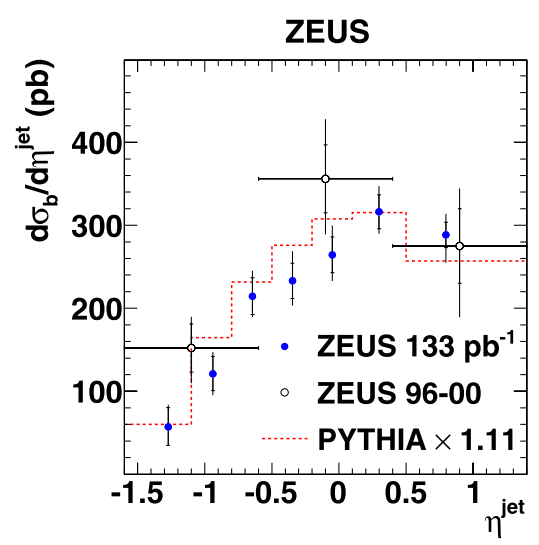

Fig. 7 Differential beauty-jet photoproduction cross sections as a function of $\eta^{\text {jet }}$. The filled circles show the results from this analysis (the same data as shown in Fig. 6c); the open circles show the results from a previously published measurement [3]. The inner error bars are the statistical uncertainties, while the outer error bars show the statistical and systematic uncertainties added in quadrature. The scaled PYTHIA MC prediction is also shown (dashed line) $b$-quark pseudorapidity in the laboratory frame, $\left|\eta_{b}\right|<2$, for $Q^{2}<1 \mathrm{GeV}^{2}$ and $0.2<y<0.8$. The $\bar{b}$ quark was not taken into account in the definition of the $b$-quark cross section. The measurement presented here extends the kinematic region to higher $p_{\mathrm{T}}^{b}$ values than previous measurements and represents the most precise measurement of $b$-quark photoproduction at HERA. Good agreement with the NLO QCD prediction is observed for many independent ZEUS measurements, giving a consistent picture of $b$-quark photoproduction over a wide range of $p_{\mathrm{T}}^{b}$.

The corresponding $c$-quark cross sections were also calculated and are shown in Fig. 9. Due to the lower mass of the charm quark, its momentum is more affected by gluon radiation. Hence the corresponding cross section is shown as a function of the parton-level jet momentum (calculated as in Sect. 7) rather than that of the quark. Here the cross sections have been extrapolated to the region $\left|\eta_{c \text {-jet }}\right|<1.5$, as it corresponded better to the measurements.

The $c$-quark jet cross sections are consistent with previous ZEUS measurements $[8,11]$ and are in good agreement with the NLO QCD prediction.
Fig. 8 a Summary of differential cross sections for $b$-quark production as a function of $p_{\mathrm{T}}^{b}$ as measured by the ZEUS collaboration. The measurements are shown as points, with the results of this analysis shown as inverted triangles. The inner error bars are the statistical uncertainties, while the outer error bars show the statistical and systematic uncertainties added in quadrature. The band represents the NLO QCD prediction and its theoretical uncertainty. The solid line shows the prediction for $\mu^{2}=\left(m_{b}^{2}+p_{\mathrm{T}}^{2}\right) / 4$, while the dashed line shows the prediction for $\mu^{2}=m_{b}^{2}+p_{\mathrm{T}}^{2}$. b The ratio of the measured cross sections, $\sigma^{\text {meas }}$, to the theoretical prediction, $\sigma^{\text {th }}$, for $\mu^{2}=\left(m_{b}^{2}+p_{\mathrm{T}}^{2}\right) / 4$

\section{ZEUS}

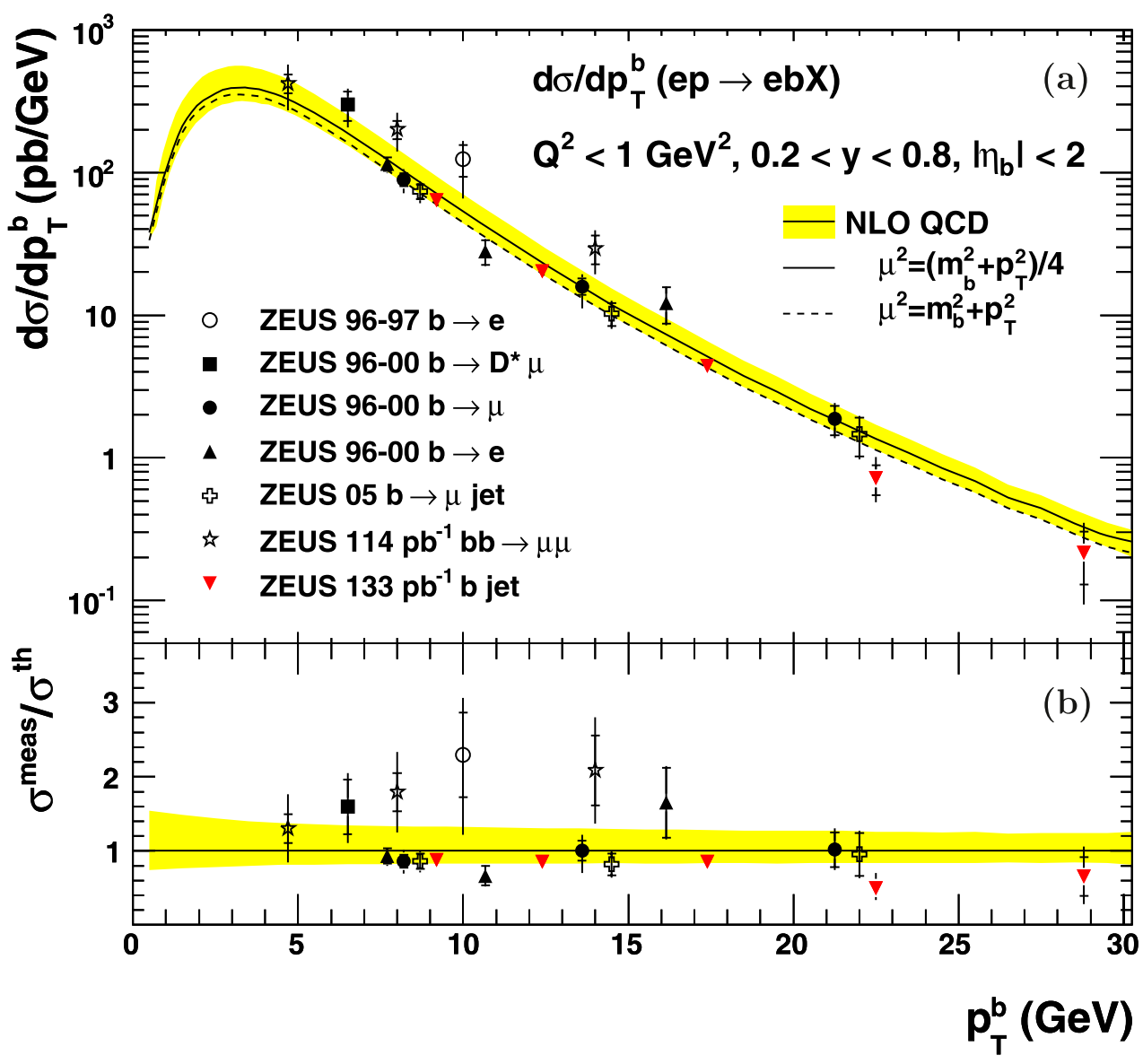


Fig. 9 a Summary of differential cross sections for $c$-quark jet production as a function of $p_{\mathrm{T}}^{c \text {-jet }}$ as measured by the ZEUS collaboration. The measurements are shown as points, with the results of this analysis shown as inverted triangles. The inner error bars are the statistical uncertainties, while the outer error bars show the statistical and systematic uncertainties added in quadrature. The band represents the NLO QCD prediction and its theoretical uncertainty. The solid line shows the prediction for $\mu^{2}=\left(m_{c}^{2}+p_{\mathrm{T}}^{2}\right) / 4$, while the dashed line shows the prediction for $\mu^{2}=m_{c}^{2}+p_{\mathrm{T}}^{2}$. b The ratio of the measured cross sections, $\sigma^{\text {meas }}$, to the theoretical prediction, $\sigma^{\text {th }}$, for $\mu^{2}=\left(m_{c}^{2}+p_{\mathrm{T}}^{2}\right) / 4$
ZEUS

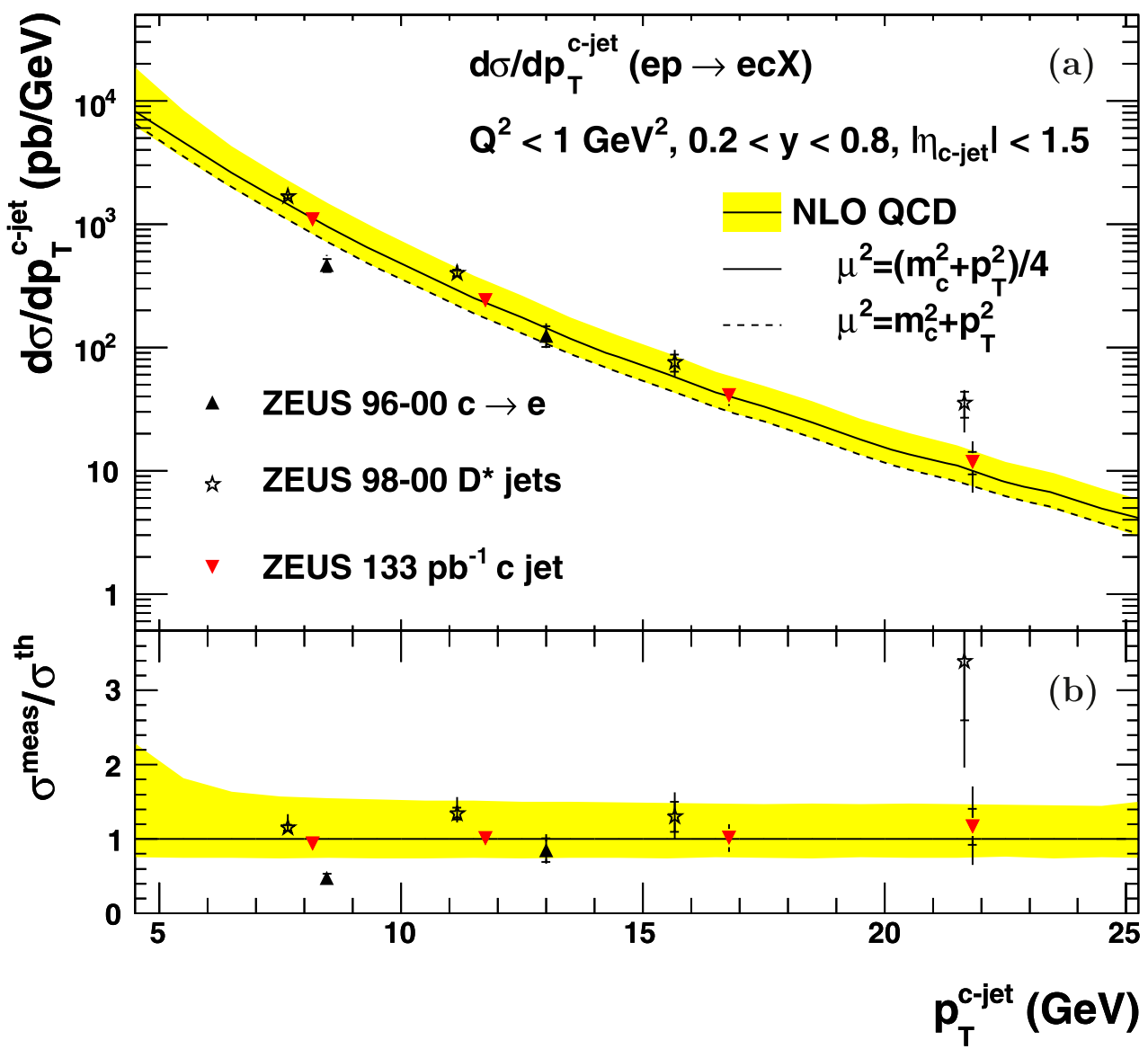

\section{Conclusions}

Inclusive beauty- and charm-jet cross sections in photoproduction at HERA have been presented, exploiting the long lifetimes and large masses of $b$ and $c$ hadrons. Compared to previous measurements of specific decay chains, this analysis has substantially increased statistics and a reduced dependence on the branching fractions. The background from light-quark jets was suppressed by using the subtracted decay-length significance distribution of secondary vertices.

The visible cross sections as well as differential cross sections as a function of $p_{\mathrm{T}}^{\text {jet }}$ and $\eta^{\text {jet }}$ have been compared with NLO QCD calculations. Good agreement is observed.

In order to be able to compare these cross sections with others, they have been extrapolated to the region $\left|\eta_{b}\right|<2$ $\left(\left|\eta_{c \text {-jet }}\right|<1.5\right)$ using the NLO QCD predictions. Cross sections as a function of the transverse momentum of the $b$ quark and of the $c$-quark jet have been determined and compared with previous ZEUS measurements. The measurements agree with each other and give a consistent picture of heavy-quark photoproduction over a wide kinematic range.

The charm cross sections presented in this paper are more precise than previous measurements made by the ZEUS col- laboration and have similar accuracy as measurements made by H1. The beauty cross sections represent the most precise measurements of $b$-quark photoproduction made at HERA.

Acknowledgements We appreciate the contributions to the construction and maintenance of the ZEUS detector of many people who are not listed as authors. The HERA machine group and the DESY computing staff are especially acknowledged for their success in providing excellent operation of the collider and the data-analysis environment. We thank the DESY directorate for their strong support and encouragement.

Open Access This article is distributed under the terms of the Creative Commons Attribution Noncommercial License which permits any noncommercial use, distribution, and reproduction in any medium, provided the original author(s) and source are credited.

\section{References}

1. S. Frixione, P. Nason, G. Ridolfi, Nucl. Phys. B 454, 3 (1995)

2. S. Frixione et al., Phys. Lett. B 348, 633 (1995)

3. S. Chekanov et al. (ZEUS Collab.), Phys. Rev. D 70, 12008 (2004)

4. S. Chekanov et al. (ZEUS Collab.), J. High Energy Phys. 02, 032 (2009) 
5. S. Chekanov et al. (ZEUS Collab.), J. High Energy Phys. 04, 133 (2009)

6. A. Aktas et al. (H1 Collab.), Eur. Phys. J. C 41, 453 (2005)

7. J. Breitweg et al. (ZEUS Collab.), Eur. Phys. J. C 18, 625 (2001)

8. S. Chekanov et al. (ZEUS Collab.), Phys. Rev. D 78, 072001 (2008)

9. A. Aktas et al. (H1 Collab.), Eur. Phys. J. C 47, 597 (2006)

10. J. Breitweg et al. (ZEUS Collab.), Eur. Phys. J. C 6, 67 (1999)

11. S. Chekanov et al. (ZEUS Collab.), Nucl. Phys. B 729, 492 (2005)

12. S. Chekanov et al. (ZEUS Collab.), Eur. Phys. J. C 44, 351 (2005)

13. S. Chekanov et al. (ZEUS Collab.), Eur. Phys. J. C 50, 299 (2007)

14. S. Chekanov et al. (ZEUS Collab.), Phys. Lett. B 565, 87 (2003)

15. A. Aktas et al. (H1 Collab.), Eur. Phys. J. C 50, 251 (2006)

16. A. Aktas et al. (H1 Collab.), Phys. Lett. B 621, 56 (2005)

17. A. Polini et al., Nucl. Instrum. Methods Phys. Res. A 581, 656 (2007)

18. U. Holm (ed.) (ZEUS Collab.), The ZEUS Detector. Status Report (unpublished), DESY, 1993, available on http://www-zeus.desy. de/bluebook/bluebook.html

19. N. Harnew et al., Nucl. Instrum. Methods Phys. Res. A 279, 290 (1989)

20. B. Foster et al., Nucl. Phys. B, Proc. Suppl. 32, 181 (1993)

21. B. Foster et al., Nucl. Instrum. Methods Phys. Res. A 338, 254 (1994)

22. M. Derrick et al., Nucl. Instrum. Methods Phys. Res. A 309, 77 (1991)

23. A. Andresen et al., Nucl. Instrum. Methods Phys. Res. A 309, 101 (1991)

24. A. Caldwell et al., Nucl. Instrum. Methods Phys. Res. A 321, 356 (1992)

25. A. Bernstein et al., Nucl. Instrum. Methods Phys. Res. A 336, 23 (1993)

26. J. Andruszków et al., Preprint DESY-92-066, DESY, 1992

27. M. Derrick et al. (ZEUS Collab.), Z. Phys. C 63, 391 (1994)

28. J. Andruszków et al., Acta Phys. Pol. B 32, 2025 (2001)

29. M. Helbich et al., Nucl. Instrum. Methods Phys. Res. A 565, 572 (2006)

30. T. Sjöstrand et al., Commun. Comput. Phys. 135, 238 (2001)

31. E. Norrbin, T. Sjöstrand, Eur. Phys. J. C 17, 137 (2000)
32. T. Sjöstrand, L. Lönnblad, S. Mrenna, Preprint hep-ph/0108264, 2001

33. V. Schönberg, Ph.D. Thesis, Universität Bonn, Bonn, Germany, Report BONN-IR-2010-05, 2010, available on http://hss.ulb. uni-bonn.de/diss_online

34. H.L. Lai et al., Phys. Rev. D 55, 1280 (1997)

35. H.L. Lai et al., Eur. Phys. J. C 12, 375 (2000)

36. M. Glück, E. Reya, A. Vogt, Phys. Rev. D 46, 1973 (1992)

37. M. Glück, E. Reya, A. Vogt, Phys. Rev. D 45, 3986 (1992)

38. Particle Data Group, C. Amsler et al., Phys. Lett. B 667, 1 (2008)

39. R. Brun et al., geant3. Technical Report CERN-DD/EE/84-1, CERN, 1987

40. P.D. Allfrey et al., Nucl. Instrum. Methods Phys. Res. A 580, 1257 (2007)

41. W.H. Smith, K. Tokushuku, L.W. Wiggers, in Proc. Computing in High-Energy Physics (CHEP), ed. by C. Verkerk, W. Wojcik, Annecy, France, (CERN, Geneva, 1992), p. 222. Also in preprint DESY 92-150B

42. G.M. Briskin, Ph.D. Thesis, Tel Aviv University, Report DESYTHESIS 1998-036, 1998

43. S. Catani et al., Nucl. Phys. B 406, 187 (1993)

44. S.D. Ellis, D.E. Soper, Phys. Rev. D 48, 3160 (1993)

45. F. Jacquet, A. Blondel, in Proceedings of the Study for an ep Facility for Europe, ed. by U. Amaldi, Hamburg, Germany (1979), p. 391. Also in preprint DESY 79/48

46. K. Rose, E. Gurewitz, G.C. Fox, Phys. Rev. Lett. 65, 945 (1990)

47. K. Rose, Proc. IEEE 86, 2210-2239 (1998)

48. F. Didierjean, G. Duchêne, A. Lopez-Martens, Nucl. Instrum. Methods 615, 188 (2010)

49. A. Yagües, Ph.D. Thesis, Humboldt Universität zu Berlin, Berlin, Germany, 2008, available on http://edoc.hu-berlin.de

50. S. Chekanov et al. (ZEUS Collab.), J. High Energy Phys. 04, 082 (2009)

51. C. Peterson et al., Phys. Rev. D 27, 105 (1983)

52. P. Nason, C. Oleari, Nucl. Phys. B 565, 245 (2000)

53. S. Frixione et al., Nucl. Phys. B 412, 225 (1994)

54. J. Pumplin et al., J. High Energy Phys. 07, 012 (2002)

55. S. Chekanov et al. (ZEUS Collab.), Phys. Rev. D 70, 12008 (2004) 